\title{
Krein Systems and Canonical Systems on a Finite Interval: Accelerants with a Jump Discontinuity at the Origin and Continuous Potentials
}

\author{
D. Alpay, I. Gohberg (Z"L), M. A. Kaashoek, \\ L. Lerer and A. L. Sakhnovich
}

\begin{abstract}
This paper is devoted to connections between accelerants and potentials of Krein systems and of canonical systems of Dirac type, both on a finite interval. It is shown that a continuous potential is always generated by an accelerant, provided the latter is continuous with a possible jump discontinuity at the origin. Moreover, the generating accelerant is uniquely determined by the potential. The results are illustrated on pseudo-exponential potentials. The paper is a continuation of the earlier paper of the authors (Alpay et al. in Modern Analysis and Applications. The Mark Krein Centenary Conference, vol. 2, pp. 19-36, OT 191. Birkhäuser, Basel, 2009) dealing with the direct problem for Krein systems.
\end{abstract}

Mathematics Subject Classification (2010). Primary 34A55, 45D05; Secondary 47A45, 47B35, 93B15.

Keywords. Krein systems, canonical systems, accelerant, continuous potential, pseudo-exponential potential, fundamental solution, inverse problems, semi-separable integral operators, triangular operators.

When Israel Gohberg, the second author of this paper, passed away on October 12, 2009, the work on this paper was finished, except for the last section of which only the first draft existed. The expression $\mathrm{Z}^{\prime \prime} \mathrm{L}$ after his name is used in Hebrew and means "of blessed memory".

D. Alpay wishes to thank the Earl Katz family for endowing the chair which supported his research. The work of Leonid Lerer was supported by ISF-Israel Science Foundation, Grant No 121/09, and that of Alexander Sakhnovich by the Austrian Science Fund (FWF) under Grant No. Y330. 


\section{Introduction}

Let $\mathbf{T}>0$, and let $k$ be a scalar continuous function on the interval $[-\mathbf{T}, \mathbf{T}]$ which is hermitian, that is, $k(-t)=\overline{k(t)}$ on $-\mathbf{T} \leq t \leq \mathbf{T}$. Assume that for each $0<\tau \leq \mathbf{T}$ the corresponding convolution integral operator $T_{\tau}$ on $L^{2}(0, \tau)$,

$$
\left(T_{\tau} f\right)(t)=f(t)-\int_{0}^{\tau} k(t-s) f(s) d s, \quad 0 \leq t \leq \tau,
$$

is invertible, and let $\gamma_{\tau}(t, s)$ be the corresponding resolvent kernel, i.e.,

$$
\gamma_{\tau}(t, s)-\int_{0}^{\tau} k(t-\xi) \gamma_{\tau}(\xi, s) d \xi=k(t-s), \quad 0 \leq t, s \leq \tau .
$$

Consider the entire functions

$$
\begin{aligned}
& \mathcal{P}(\tau, \lambda)=e^{i \lambda \tau}\left(1+\int_{0}^{\tau} e^{-i \lambda x} \gamma_{\tau}(x, 0) d x\right), \\
& \mathcal{P}_{*}(\tau, \lambda)=1+\int_{0}^{\tau} e^{i \lambda x} \gamma_{\tau}(\tau-x, \tau) d x,
\end{aligned}
$$

and put $Y(\tau, \lambda)=\left[\mathcal{P}(\tau, \lambda) \mathcal{P}_{*}(\tau, \lambda)\right]$. Then, as was proved by M.G. Krein in [8], the function $Y(\tau, \lambda)$ satisfies the differential system

$$
\frac{\partial}{\partial \tau} Y(\tau, \lambda)=Y(\tau, \lambda)\left(i \lambda\left[\begin{array}{ll}
1 & 0 \\
0 & 0
\end{array}\right]+\left[\begin{array}{cc}
0 & a(\tau) \\
a(\tau) & 0
\end{array}\right]\right)
$$

with $a(\tau)=\gamma_{\tau}(\tau, 0)$ for $\tau \in(0, \mathbf{T}]$. The functions $\mathcal{P}(\tau, \lambda)$ and $\mathcal{P}_{*}(\tau, \lambda)$ are usually referred to as Krein orthogonal functions.

We call (1.5) a Krein system when, as in the previous paragraph, the function $a$ is given by $a(\tau)=\gamma_{\tau}(\tau, 0)$, where $\gamma_{\tau}(t, s)$ is the resolvent kernel corresponding to some $k$ on $[-\mathbf{T}, \mathbf{T}]$ with the properties described in the previous paragraph. In that case, following Krein, the function $k$ is called an accelerant for (1.5), and we shall refer to $a$ as the potential associated with the accelerant $k$.

The result referred to above holds in greater generality, namely for systems with matrix-valued accelerants that are allowed to have a jump discontinuity at the origin. In fact, in [1] the following result is proved.

Theorem 1.1. Let $k$ be an $r \times r$-matrix function, which is hermitian, i.e., $k(-t)=k(t)^{*}$, and continuous on $-\mathbf{T} \leq t \leq \mathbf{T}$ with possibly a jump discontinuity at the origin. Assume that for each $0<\tau \leq \mathbf{T}$ the corresponding integral operator $T_{\tau}$ on $L_{r}^{2}(0, \tau)$ given by (1.1) is invertible, and let $\gamma_{\tau}(t, s)$ be the corresponding resolvent kernel as in (1.2). Put 


$$
\begin{aligned}
& \mathcal{P}(\tau, \lambda)=e^{i \lambda \tau}\left(I_{r}+\int_{0}^{\tau} e^{-i \lambda x} \gamma_{\tau}(x, 0) d x\right) \\
& \mathcal{P}_{*}(\tau, \lambda)=I_{r}+\int_{0}^{\tau} e^{i \lambda x} \gamma_{\tau}(\tau-x, \tau) d x .
\end{aligned}
$$

Then $a(\tau)=\gamma_{\tau}(0, \tau)$, with $0<\tau \leq \mathbf{T}$, extends to a continuous function on $[0, \mathbf{T}]$ and $Y(\tau, \lambda)=\left[\mathcal{P}(\tau, \lambda) \mathcal{P}_{*}(\tau, \lambda)\right]$ satisfies

$$
\frac{\partial}{\partial \tau} Y(\tau, \lambda)=Y(\tau, \lambda)\left(i \lambda\left[\begin{array}{cc}
I_{r} & 0 \\
0 & 0
\end{array}\right]+\left[\begin{array}{cc}
0 & a(\tau) \\
a(\tau)^{*} & 0
\end{array}\right]\right) .
$$

The phrase $k$ is continuous on $-\mathbf{T} \leq t \leq \mathbf{T}$ with possibly a jump discontinuity at the origin, which appears in the above theorem, means that the function $k$ is continuous on the intervals $-\mathbf{T} \leq t<0$ and $0<t \leq \mathbf{T}$ and that the two limits $\lim _{t \downarrow 0} k(t)$ and $\lim _{t \uparrow 0} k(t)$ exist. The actual value of $k$ at the origin does not play a role.

From [6] we know that under the conditions in Theorem 1.1 the resolvent kernel function $\gamma_{\tau}(t, s)$ is continuous on the triangles on $0 \leq s<t \leq \tau$ and $0 \leq t<s \leq \tau$, and that $\gamma_{\tau}(t, s)$ has a continuous extension on the closures of each of these triangles. Jumps may appear on the diagonal $0 \leq s=x \leq \tau$. In particular, the evaluation of $\gamma_{\tau}$ at the point $(\tau, 0)$, appearing in Theorem 1.1, is well-defined.

As for the scalar case we call (1.8) a Krein system when the potential $a$ is obtained in the way described in Theorem 1.1, and in that case we say that $k$ is an accelerant for (1.8).

In this paper we deal, among other things, with the following inverse problem. Consider the system (1.8) and assume that the potential $a$ is an $r \times r$-matrix valued function continuous on $[0, \mathbf{T}]$. Does it follow that (1.8) is a Krein system? In other words, does there exist an $r \times r$-matrix valued accelerant $k$ on $[-\mathbf{T}, \mathbf{T}]$, with possibly a jump discontinuity at the origin, such that the potential corresponding to $k$ is the given potential $a$ ? If we restrict to continuous accelerants, the answer is negative. For instance (see [1]), the potential

$$
a(\tau)=\frac{2 i}{1+e^{-2 i \tau}}, \quad \tau \in[0,1],
$$

does not have a continuous accelerant. However, we shall prove that for the larger class of accelerants introduced here, the answer is affirmative.

Krein systems are closely related to canonical differential systems of Dirac type. In fact, if $Y$ is a $\mathbb{C}^{2 r \times 2 r}$-valued solution of the system (1.8) with potential $a$, then the function

$$
U(\tau, \lambda)=e^{-i \tau \lambda} Y(\tau,-2 \bar{\lambda})^{*}
$$

is a solution of the canonical system

$$
-i j \frac{\mathrm{d}}{d \tau} U(\tau, \lambda)=\lambda U(\tau, \lambda)+\left[\begin{array}{cc}
0 & v(\tau) \\
v(\tau)^{*} & 0
\end{array}\right] U(\tau, \lambda),
$$


where

$$
j=\left[\begin{array}{cc}
I_{r} & 0 \\
0 & -I_{r}
\end{array}\right] \quad \text { and } \quad v(\tau)=-i a(\tau) \quad(0 \leq \tau \leq \mathbf{T}) .
$$

It will be convenient to state our main results in terms of a canonical system rather than a Krein system.

In this paper we show that a continuous matrix-valued potential $v$ is always generated by an accelerant (provided a jump discontinuity at the origin is allowed) and that an accelerant is uniquely determined by the potential, that is, if two accelerants generate the same potential, then they are equal. In fact, we shall prove the following theorem.

Theorem 1.2. Consider the canonical system (1.10), and assume that its potential $v$ is continuous on the interval $[0, \mathbf{T}]$. Then, there is a unique $r \times r$ matrix function $k$, which is hermitian, i.e., $k(-t)=k(t)^{*}$, and continuous on $-\mathbf{T} \leq t \leq \mathbf{T}$ with possibly a jump discontinuity at the origin, such that the following holds: for each $0<\tau \leq \mathbf{T}$ the convolution operator

$$
\left(T_{\tau} f\right)(t)=f(t)-\int_{0}^{\tau} k(t-s) f(s) d s, \quad 0 \leq t \leq \tau,
$$

is invertible on $L_{r}^{2}(0, \tau)$, and the potential $v$ of $(1.10)$ is given by

$$
v(\tau)=-i \gamma_{\tau}(\tau, 0), \quad 0<\tau \leq \mathbf{T} .
$$

Here $\gamma_{\tau}(t, s)$ is the resolvent kernel corresponding to $T_{\tau}$ as in (1.2).

In analogy with the theory of Krein systems, an $r \times r$ matrix function $k$ with the properties described in the above theorem will be called an accelerant for the canonical system (1.10). In this case we also say that the potential $v$ is generated by the accelerant $k$. Using this terminology, Theorem 1.2 just tells us that a canonical system with a continuous potential has a unique accelerant.

Given a continuous matrix-valued potential $v$ we shall also present a formula for the fundamental solution of the canonical system (1.10) in terms of the accelerant generating the potential $v$. The result (Theorem 2.1 in Sect. 2) can be viewed as an addition to Theorem 1.1

The statement in Theorem 1.2 about the uniqueness of the accelerant is known and has been proved in [1] using recent results about the continuous analogue of the resultant for certain entire matrix functions (see Theorem 1.3 in [1] for further details). In this paper we give a new proof of the uniqueness using a formula for the fundamental solution of the canonical system (1.10) in terms of a given accelerant, which is presented in Theorem 2.1.

For the case when the potential $v$ is bounded, bounded accelerants $k$ have been constructed in [10] following the scheme outlined in Section 8.2 of [15] (see also [9]). In the present paper, to prove Theorem 1.2, the approach of [10] is specified and developed further for the case of continuous potentials. Also the material related to Theorem 2.1 below is inspired by and builds on results from Sections 3 and 4 in [10]. 
The paper consists of six sections including this introduction. In Sect. 2 we derive a formula for the fundamental solution of the canonical system (1.10) in terms of its accelerant. The result is used in in Sect. 3 to give a new proof of the uniqueness of the accelerant given the potential as stated in Theorem 1.2. The next two sections complete the proof of Theorem 1.2. Section 4 has an auxiliary character and is interesting in its own right. We show that a lower triangular semi-separable integral operator from a certain class is similar to the operator of integration and that the corresponding similarity operator can be chosen in such a way that both this similarity operator and its inverse map functions with a continuous derivative into functions with a continuous derivative. This result is then used in Sect. 5 to prove Theorem 1.2. In the final section the main result of Sect. 2 is specified further for pseudo-exponential potentials.

\section{The Fundamental Solution}

Throughout this section $k$ is a $r \times r$ matrix function on $[-\mathbf{T}, \mathbf{T}]$, which is hermitian, i.e. $k(-t)=k(t)^{*}$, and $k$ is continuous on $-\mathbf{T} \leq t \leq \mathbf{T}$ with possibly a jump discontinuity at the origin. We assume that $k$ is an accelerant for the canonical system (1.10). The latter means that for each $0<\tau \leq \mathbf{T}$ the convolution operator (1.1) is invertible on $L_{r}^{2}(0, \tau)$, and the potential $v$ is the $r \times r$ continuous matrix function on $[0, \mathbf{T}]$ determined by $k$ via the formula

$$
v(\tau)=-i \gamma_{\tau}(\tau, 0), \quad 0<\tau \leq \mathbf{T},
$$

where $\gamma_{\tau}(t, s)$ is the corresponding resolvent kernel as in (1.2).

We shall derive (explicitly in terms of the accelerant $k$ ) the fundamental solution $u(x, \lambda)$ of $(1.10)$ satisfying the initial condition

$$
u(0, \lambda)=Q^{*}, \quad \text { where } Q=\frac{1}{\sqrt{2}}\left[\begin{array}{cc}
I_{r} & -I_{r} \\
I_{r} & I_{r}
\end{array}\right] .
$$

For this purpose we need the following $r \times r$ matrix functions:

$$
\ell_{1}(x, \lambda)=e^{2 i \lambda x}\left(I_{r}-2 \int_{0}^{x} e^{-2 i \lambda t} k(t) d t\right), \quad \ell_{2}(x, \lambda)=e^{2 i \lambda x} I_{r} .
$$

Both $\ell_{1}(\cdot, \lambda)$ and $\ell_{2}(\cdot, \lambda)$ are defined on $[0, \mathbf{T}]$. Note that for $0 \leq x \leq \mathbf{T}$ we have

$$
\frac{d}{d x} \ell_{1}(x, \lambda)=2 i \lambda \ell_{1}(x, \lambda)-2 k(x), \quad \frac{d}{d x} \ell_{2}(x, \lambda)=2 i \lambda \ell_{2}(x, \lambda) .
$$

The next theorem is the main result of this section.

Theorem 2.1. Assume that the $r \times r$ matrix function $k$ is an accelerant for the canonical system (1.10), and let $\gamma_{\tau}(t, s)$ be the corresponding resolvent kernel as in (1.2). For $0 \leq \tau \leq \mathbf{T}, \lambda \in \mathbb{C}$, and $j=1,2$ put 


$$
\begin{aligned}
& \theta_{j}(\tau, \lambda)=\frac{1}{\sqrt{2}} e^{-i \tau \lambda}\left\{\ell_{j}(\tau, \lambda)+\int_{0}^{\tau} \gamma_{\tau}(\tau, s) \ell_{j}(s, \lambda) d s\right\} \\
& \omega_{j}(\tau, \lambda)=\frac{1}{\sqrt{2}} e^{-i \tau \lambda}\left\{(-1)^{j} I_{r}+\int_{0}^{\tau} \gamma_{\tau}(0, s) \ell_{j}(s, \lambda) d s\right\},
\end{aligned}
$$

where $\ell_{1}(\cdot, \lambda)$ and $\ell_{2}(\cdot, \lambda)$ are given by (2.3). Then the $2 r \times 2 r$ matrix function $u(\tau, \lambda)$ defined by

$$
u(\tau, \lambda)=\left[\begin{array}{ll}
\theta_{1}(\tau, \lambda) & \theta_{2}(\tau, \lambda) \\
\omega_{1}(\tau, \lambda) & \omega_{2}(\tau, \lambda)
\end{array}\right], \quad 0 \leq \tau \leq \mathbf{T},
$$

is the fundamental solution of (1.10) with initial condition (2.2).

Using the definition of $\ell_{2}(\cdot, \lambda)$ in the second part of $(2.3)$ we see that

$$
\begin{aligned}
& \theta_{2}(\tau, \lambda)=\frac{1}{\sqrt{2}} e^{i \tau \lambda}\left(I_{r}+\int_{0}^{\tau} e^{-2 i \lambda s} \gamma_{\tau}(\tau, \tau-s) d s\right), \\
& \omega_{2}(\tau, \lambda)=\frac{1}{\sqrt{2}} e^{-i \tau \lambda}\left(I_{r}+\int_{0}^{\tau} e^{2 i \lambda s} \gamma_{\tau}(0, s) d s\right) .
\end{aligned}
$$

The formula for $\omega_{2}(\tau, \lambda)$ is immediate from the definition and the one for $\theta_{2}(\tau, \lambda)$ follows using the following calculation:

$$
\begin{aligned}
\theta_{2}(\tau, \lambda) & =\frac{1}{\sqrt{2}} e^{-i \tau \lambda}\left(e^{2 i \lambda \tau} I_{r}+\int_{0}^{\tau} e^{2 i \lambda s} \gamma_{\tau}(\tau, s) d s\right) \\
& =\frac{1}{\sqrt{2}} e^{-i \tau \lambda}\left(e^{2 i \lambda \tau} I_{r}+\int_{0}^{\tau} e^{2 i \lambda(\tau-s)} \gamma_{\tau}(\tau, \tau-s) d s\right) \\
& =\frac{1}{\sqrt{2}} e^{i \tau \lambda}\left(I_{r}+\int_{0}^{\tau} e^{-2 i \lambda s} \gamma_{\tau}(\tau, \tau-s) d s\right) .
\end{aligned}
$$

The expressions (2.8) and (2.9) show that the functions $\theta_{2}(\tau, \lambda)$ and $\omega_{2}(\tau, \lambda)$ are closely related to the Krein orthogonal entire matrix functions $\mathcal{P}(\tau, \lambda)$ and $\mathcal{P}_{*}(\tau, \lambda)$ appearing in Theorem 1.1. In fact we have

$$
\theta_{2}(\tau, \lambda)=\frac{1}{\sqrt{2}} e^{-i \tau \lambda} \mathcal{P}_{*}(\tau, 2 \bar{\lambda})^{*}, \quad \omega_{2}(\tau, \lambda)=\frac{1}{\sqrt{2}} e^{i \tau \lambda} \mathcal{P}(\tau, 2 \bar{\lambda})^{*}
$$

Thus Theorem 2.1 can be seen as an addition to Theorem 1.1.

To prove Theorem 2.1, we need some preliminaries. In the sequel we write $T$ in place of $T_{\mathbf{T}}$. The fact that $T_{\tau}$ is invertible for each $0<\tau \leq \mathbf{T}$ is equivalent to $T$ being strictly positive. The latter property implies that $T$ factorizes as $T=\Gamma \Gamma^{*}$, where $\Gamma$ is an invertible lower triangular integral operator, 


$$
\begin{gathered}
(\Gamma f)(x)=f(x)+\int_{0}^{x} \gamma_{-}(x, t) f(s) d s, \quad 0 \leq x \leq \mathbf{T}, \\
\left(\Gamma^{-1} f\right)(x)=f(x)+\int_{0}^{x} \gamma_{-}^{\times}(x, s) f(s) d s, \quad 0 \leq x \leq \mathbf{T},
\end{gathered}
$$

with both $\gamma_{-}(x, s)$ and $\gamma_{-}^{\times}(x, s)$ being continuous on $0 \leq s \leq x \leq \mathbf{T}$. We shall refer to $T=\Gamma \Gamma^{*}$ as the $L U$-factorization of $T$. From [6] we also know that

$$
\gamma_{-}^{\times}(\tau, s)=\gamma_{\tau}(\tau, s), \quad 0 \leq s \leq \tau \leq \mathbf{T} .
$$

We shall need the following three lemmas.

Lemma 2.2. We have

$$
\left(\Gamma^{-1} k\right)(\tau)=\left(T_{\tau}^{-1}\left(\left.k\right|_{[0, \tau]}\right)\right)(\tau)=\gamma_{\tau}(\tau, 0), \quad 0<\tau \leq \mathbf{T} .
$$

Proof. We first prove the second equality in (2.13). Since $\gamma_{\tau}(t, s)$ is the resolvent kernel corresponding to $T_{\tau}$, we know (cf., (1.2)) that

$$
\gamma_{\tau}(t, s)-\int_{0}^{\tau} k(t-\xi) \gamma_{\tau}(\xi, s) d \xi=k(t-s), \quad 0 \leq s \leq t \leq \tau .
$$

This equality holds a.e on $0 \leq s \leq t \leq \tau$. But then, since both $\gamma_{\tau}(t, s)$ and $k(t-s)$ are continuous on $0 \leq s \leq t \leq \tau$, the above equality holds at each point of $0 \leq s \leq t \leq \tau$. In particular, at the point $(t, 0)$. Thus

$$
\gamma_{\tau}(t, 0)-\int_{0}^{\tau} k(t-\alpha) \gamma_{\tau}(\alpha, 0) d \alpha=k(t), \quad 0 \leq t \leq \tau .
$$

This shows that $T_{\tau} \gamma_{\tau}(\cdot, 0)=\left.k\right|_{[0, \tau]}$, and hence

$$
\left(T_{\tau}^{-1}\left(\left.k\right|_{[0, \tau]}\right)\right)(x)=\gamma_{\tau}(x, 0), \quad 0 \leq x \leq \tau .
$$

For $x=\tau$ this yields the second identity in (2.13).

Next we prove the first identity in $(2.13)$. Fix $0<\tau \leq \mathbf{T}$. Since $k$ is continuous on $[0, \mathbf{T}]$, the function $\Gamma^{-1} k$ is continuous on $[0, \mathbf{T}]$. From the previous part of the proof we know that $T_{\tau}^{-1}\left(\left.k\right|_{[0, \tau]}\right)$ is continuous on $[0, \tau]$. Hence for both functions the evaluation at $\tau$ is well-defined. Moreover,

$$
\begin{aligned}
\left(\Gamma^{-1} k\right)(\tau) & =k(\tau)+\int_{0}^{\tau} \gamma_{-}^{\times}(\tau, s) k(s) d s, \\
\left(T_{\tau}^{-1}\left(\left.k\right|_{[0, \tau]}\right)(\tau)\right. & =k(\tau)+\int_{0}^{\tau} \gamma_{\tau}(\tau, s) k(s) d s .
\end{aligned}
$$

According to (2.12) we have $\gamma_{-}^{\times}(\tau, s)=\gamma_{\tau}(\tau, s)$ for $0 \leq s \leq \tau$, which yields the first equality in (2.13). 
Lemma 2.3. Let $f$ be a continuously differentiable $\mathbb{C}^{r \times m}$-valued function on the interval $[0, \mathbf{T}]$. Then $\Gamma^{-1} f$ is also continuously differentiable on $[0, \mathbf{T}]$ and

$$
\begin{aligned}
& \left(\frac{d}{d \tau} \Gamma^{-1} f\right)(\tau)-\left(\Gamma^{-1} \frac{d}{d \tau} f\right)(\tau) \\
& \quad=\gamma_{\tau}(\tau, 0)\left(f(0)+\int_{0}^{\tau} \gamma_{\tau}(0, s) f(s) d s\right), \quad 0<\tau \leq \mathbf{T} .
\end{aligned}
$$

Proof. Recall that

$$
\left(\Gamma^{-1} f\right)(\tau)=f(\tau)+\int_{0}^{\tau} \gamma_{-}^{\times}(\tau, s) f(s) d s=f(\tau)+\int_{0}^{\tau} \gamma_{\tau}(\tau, s) f(s) d s .
$$

Next, using the generalized Krein-Sobolev identities in formulas (2.10) and (2.11) of [1], we have

$$
\begin{aligned}
\frac{d}{d \tau} \int_{0}^{\tau} \gamma_{\tau}(\tau, s) f(s) d s & =\frac{d}{d \tau} \int_{0}^{\tau} \gamma_{\tau}(\tau, \tau-s) f(\tau-s) d s \\
& =\gamma_{\tau}(\tau, 0) f(0)+(\alpha),
\end{aligned}
$$

where

$$
\begin{aligned}
(\alpha)= & \int_{0}^{\tau} \frac{d}{d \tau}\left(\gamma_{\tau}(\tau, \tau-s) f(\tau-s)\right) d s \\
= & \int_{0}^{\tau} \gamma_{\tau}(\tau, 0) \gamma_{\tau}(0, \tau-s) f(\tau-s) d s \\
& +\int_{0}^{\tau} \gamma_{\tau}(\tau, \tau-s)\left(\frac{d}{d \tau} f\right)(\tau-s) d s .
\end{aligned}
$$

We conclude that

$$
\begin{aligned}
\left(\frac{d}{d \tau} \Gamma^{-1} f\right)(\tau)= & \left(\frac{d}{d \tau} f\right)(\tau)+\int_{0}^{\tau} \gamma_{\tau}(\tau, s)\left(\frac{d}{d \tau} f\right)(s) d s \\
& +\gamma_{\tau}(\tau, 0)\left(f(0)+\int_{0}^{\tau} \gamma_{\tau}(0, s) f(s) d s\right) \\
= & \left(\Gamma^{-1} \frac{d}{d \tau} f\right)(\tau) \\
& +\gamma_{\tau}(\tau, 0)\left(f(0)+\int_{0}^{\tau} \gamma_{\tau}(0, s) f(s) d s\right) .
\end{aligned}
$$

Thus $\Gamma^{-1} f$ is continuously differentiable and (2.15) holds. 
Lemma 2.4. For each $g \in L_{r}^{2}(0, \mathbf{T})$ we have

$$
\int_{0}^{\tau}\left(\Gamma^{-1} k\right)(t)^{*}\left(\Gamma^{-1} g\right)(t) d t=\int_{0}^{\tau} \gamma_{\tau}(0, t) g(t) d t, \quad 0 \leq \tau \leq \mathbf{T} .
$$

Proof. The case when $\tau=0$ is trivial. Fix $0<\tau \leq \mathbf{T}$. Let $\Pi_{\tau}$ be the projection of $L_{r}^{2}(0, \mathbf{T})$ onto $L_{r}^{2}(0, \tau)$ defined by $\Pi_{\tau} f=\left.f\right|_{[0, \tau]}$. Note that $\Pi_{\tau}^{*}$ is the canonical embedding of $L_{r}^{2}(0, \tau)$ into $L_{r}^{2}(0, \mathbf{T})$. Put $P_{\tau}=\Pi_{\tau}^{*} \Pi_{\tau}$. Then $P_{\tau}$ is the orthogonal projection of $L_{r}^{2}(0, \mathbf{T})$ onto the subspace consisting of all functions in $L_{r}^{2}(0, \mathbf{T})$ with support in $[0, \tau]$. Since $\Gamma$ and $\Gamma^{-1}$ are lower triangular, we have

$$
\Pi_{\tau} \Gamma=\Pi_{\tau} \Gamma P_{\tau}, \quad \Pi_{\tau} \Gamma^{-1}=\Pi_{\tau} \Gamma^{-1} P_{\tau} .
$$

From the second identity in (2.17), the definition of $T_{\tau}$, and the factorization $T_{\mathbf{T}}=\Gamma \Gamma^{*}$, we see that

$$
T_{\tau}=\Pi_{\tau} T_{\mathbf{T}} \Pi_{\tau}^{*}=\Pi_{\tau} \Gamma \Gamma^{*} \Pi_{\tau}^{*}=\Pi_{\tau} \Gamma P_{\tau} \Gamma \Pi_{\tau}^{*}=\Pi_{\tau} \Gamma \Pi_{\tau}^{*} \Pi_{\tau} \Gamma^{*} \Pi_{\tau}^{*} .
$$

Since, by lower triangularity, $\Pi_{\tau} \Gamma \Pi_{\tau}^{*}=\left(\Pi_{\tau} \Gamma^{-1} \Pi_{\tau}^{*}\right)^{-1}$, we obtain

$$
T_{\tau}^{-1}=\Pi_{\tau} \Gamma^{-*} P_{\tau} \Gamma^{-1} \Pi_{\tau}^{*} .
$$

If follows that

$$
T_{\tau}^{-1}\left(\left.k\right|_{[0, \tau]}\right)=\Pi_{\tau} \Gamma^{-*} P_{\tau} \Gamma^{-1} \Pi_{\tau}^{*}\left(\Pi_{\tau} k\right)=\Pi_{\tau} \Gamma^{-*} P_{\tau} \Gamma^{-1} P_{\tau} k .
$$

Now using (2.14), the lower triangularity of $\Gamma^{-1}$, and the above identities one computes that

$$
\begin{aligned}
\int_{0}^{\tau}\left(\Gamma^{-1} k\right)(t)^{*}\left(\Gamma^{-1} f\right)(t) d t & =\int_{0}^{\mathbf{T}}\left(P_{\tau} \Gamma^{-1} k\right)(t)^{*}\left(P_{\tau} \Gamma^{-1} f\right)(t) d t \\
& =\int_{0}^{\mathbf{T}}\left(P_{\tau} \Gamma^{-1} P_{\tau} k\right)(t)^{*}\left(P_{\tau} \Gamma^{-1} P_{\tau} f\right)(t) d t \\
& =\int_{0}^{\mathbf{T}}\left(P_{\tau} \Gamma^{-*} P_{\tau} P_{\tau} \Gamma^{-1} P_{\tau} k\right)(t)^{*} f(t) d t \\
& =\int_{0}^{\mathbf{T}}\left(\Pi_{\tau}^{*} T_{\tau}^{-1} \Pi_{\tau} k\right)(t)^{*} f(t) d t \\
& =\int_{0}^{\tau}\left(T_{\tau}^{-1}\left(\left.k\right|_{[0, \tau]}\right)(t)^{*} f(t) d t=\int_{0}^{\tau} \gamma_{\tau}(0, t) f(t) d t .\right.
\end{aligned}
$$

This proves (2.16).

Using the identities (2.12) and (2.16) we see that the expressions for $\theta_{j}(\tau, \lambda)$ and $\omega_{j}(\tau, \lambda)$ in $(2.5)$ and $(2.6)$, respectively, can be rewritten as 
follows:

$$
\begin{aligned}
\theta_{j}(\tau, \lambda)= & \frac{1}{\sqrt{2}} e^{-i \tau \lambda}\left(\Gamma^{-1} \ell_{j}(\cdot, \lambda)\right)(\tau), \\
\omega_{j}(\tau, \lambda)= & \frac{1}{\sqrt{2}} e^{-i \tau \lambda} \\
& \times\left\{(-1)^{j} I_{r}+\int_{0}^{\tau}\left(\Gamma^{-1} k\right)(t)^{*}\left(\Gamma^{-1} \ell_{j}(\cdot, \lambda)\right)(t) d t\right\} .
\end{aligned}
$$

We are now ready to prove Theorem 2.1.

Proof of Theorem 2.1. We split the proof into three parts. The first part deals with the initial value condition; the two other parts concern the proof that $u(\tau, \lambda)$ satisfies the differential equation (1.10).

Part 1. Using the expressions for $\theta_{j}$ and $\omega_{j}$ in (2.20) and (2.21), respectively, and the fact that $\Gamma^{-1}$ is a lower triangular integral operator (see $(2.11)$ ), we obtain the following identities

$$
\theta_{j}(0, \lambda)=\frac{1}{\sqrt{2}} \ell_{j}(0, \lambda)=\frac{1}{\sqrt{2}} I_{r}, \quad \omega_{j}(0, \lambda)=\frac{1}{\sqrt{2}}(-1)^{j} I_{r} \quad(j=1,2) .
$$

Thus

$$
u(0, \lambda)=\left[\begin{array}{cc}
\frac{1}{\sqrt{2}} I_{r} & \frac{1}{\sqrt{2}} I_{r} \\
-\frac{1}{\sqrt{2}} I_{r} & \frac{1}{\sqrt{2}} I_{r}
\end{array}\right]=\frac{1}{\sqrt{2}}\left[\begin{array}{cc}
I_{r} & I_{r} \\
-I_{r} & I_{r}
\end{array}\right]=Q^{*} .
$$

Hence $u(\tau, \lambda)$ has the desired value at $\tau=0$.

To complete the proof it suffices to prove the following differential expressions:

$$
\begin{aligned}
\frac{d}{d \tau} \omega_{j}(\tau, \lambda) & =-i \lambda \omega_{j}(\tau, \lambda)-i v(\tau)^{*} \theta_{j}(\tau, \lambda), \quad j=1,2, \\
\frac{d}{d \tau} \theta_{j}(\tau, \lambda) & =i \lambda \theta_{j}(\tau, \lambda)+i v(\tau) \omega_{j}(\tau, \lambda), \quad j=1,2 .
\end{aligned}
$$

The first two identities will be proved in the next part and the other two in the final part.

Part 2. In this part we prove (2.22). Since $k$ is continuous on $[0, \mathbf{T}]$, the fact that the kernel function of the lower triangular integral operator $G^{-1}$ is continuous on $0 \leq s \leq x \leq \mathbf{T}$ implies that $\Gamma^{-1} k$ is also continuous on $[0, \mathbf{T}]$. Similarly, using the continuity of $\ell_{1}(\cdot, \lambda)$ and $\ell_{2}(\cdot, \lambda)$ on $[0, \mathbf{T}]$, we see that $\Gamma^{-1} \ell_{1}(\cdot, \lambda)$ and $\Gamma^{-1} \ell_{2}(\cdot, \lambda)$ are continuous on $[0, \mathbf{T}]$. Thus the functions under the integrals in the definitions of $\omega_{1}(\cdot, \lambda)$ and $\omega_{2}(\cdot, \lambda)$ are continuous. This implies that $\omega_{1}(\cdot, \lambda)$ and $\omega_{2}(\cdot, \lambda)$ are continuously differentiable and

$$
\begin{aligned}
\frac{d}{d \tau} \omega_{j}(\tau, \lambda)= & -i \lambda \omega_{j}(\tau, \lambda) \\
& +\frac{1}{\sqrt{2}} e^{-i \tau \lambda}\left(\Gamma^{-1} k\right)(\tau)^{*}\left(\Gamma^{-1} \ell_{j}(\cdot, \lambda)\right)(\tau), \quad j=1,2 .
\end{aligned}
$$


Using (2.13) and (2.1) we see that $\left(\Gamma^{-1} k\right)(\tau)=i v(\tau)$. This together with the expression of $\theta_{j}$ in (2.20) shows that (2.22) holds.

Part 3. In this part we prove (2.23). First note that

$$
\frac{d}{d \tau} \theta_{j}(\tau, \lambda)=-i \lambda \theta_{j}(\tau, \lambda)+\frac{1}{\sqrt{2}} e^{-i \tau \lambda}\left(\frac{d}{d \tau} \Gamma^{-1} \ell_{j}(\cdot, \lambda)\right)(\tau), \quad j=1,2
$$

Applying Lemma 2.3 with $f=\ell_{j}(\cdot, \lambda), j=1,2$, yields

$$
\begin{aligned}
\left(\frac{d}{d \tau} \Gamma^{-1} \ell_{j}(\cdot, \lambda)\right)(\tau)= & \left(\Gamma^{-1} \frac{d}{d \tau} \ell_{j}(\cdot, \lambda)\right)(\tau) \\
& +\gamma_{\tau}(\tau, 0)\left(\ell_{j}(0, \lambda)+\int_{0}^{\tau} \gamma_{\tau}(0, s) \ell_{j}(s, \lambda) d s\right), \\
& 0 \leq \tau \leq \mathbf{T} .
\end{aligned}
$$

Using the formula for the potential $v$ in (2.1) and the identity in (2.16) we obtain

$$
\begin{aligned}
\left(\frac{d}{d \tau} \Gamma^{-1} \ell_{j}(\cdot, \lambda)\right)(\tau)= & \left(\Gamma^{-1} \frac{d}{d \tau} \ell_{j}(\cdot, \lambda)\right)(\tau)+i v(\tau) \\
& +i v(\tau) \int_{0}^{\tau}\left(\Gamma^{-1} k\right)(t)^{*}\left(\Gamma^{-1} \ell_{j}(\cdot, \lambda)\right)(t) d t, \quad 0 \leq \tau \leq \mathbf{T} .
\end{aligned}
$$

Next we use the identities in $(2.4)$ and $\left(\Gamma^{-1} k\right)(\tau)=i v(\tau)$ to show that

$$
\begin{aligned}
& \frac{1}{\sqrt{2}} e^{-i \tau \lambda}\left(\Gamma^{-1} \frac{d}{d \tau} \ell_{1}(\cdot, \lambda)\right)(\tau) \\
& \quad=2 i \lambda \frac{1}{\sqrt{2}} e^{-i \tau \lambda}\left(\Gamma^{-1} \ell_{1}(\cdot, \lambda)\right)(\tau)-2 \frac{1}{\sqrt{2}} e^{-i \tau \lambda}\left(\Gamma^{-1} k\right)(\tau) \\
& \quad=2 i \lambda \theta_{1}(\tau, \lambda)-\sqrt{2} e^{-i \tau \lambda} i v(\tau)
\end{aligned}
$$

and

$$
\begin{aligned}
\frac{1}{\sqrt{2}} e^{-i \tau \lambda}\left(\Gamma^{-1} \frac{d}{d \tau} \ell_{2}(\cdot, \lambda)\right)(\tau) & =2 i \lambda \frac{1}{\sqrt{2}} e^{-i \tau \lambda}\left(\Gamma^{-1} \ell_{2}(\cdot, \lambda)\right)(\tau) \\
& =2 i \lambda \theta_{2}(\tau, \lambda) .
\end{aligned}
$$


Returning to (2.24), first for $j=1$ and next for $j=2$, we obtain

$$
\begin{aligned}
\frac{d}{d \tau} \theta_{1}(\tau, \lambda)= & -i \lambda \theta_{1}(\tau, \lambda)+2 i \lambda \theta_{1}(\tau, \lambda)-\sqrt{2} e^{-i \tau \lambda} i v(\tau) \\
& +i v(\tau) \frac{1}{\sqrt{2}} e^{-i \tau \lambda}\left(I_{r}+\int_{0}^{\tau}\left(\Gamma^{-1} k\right)(t)^{*}\left(\Gamma^{-1} \ell_{1}(\cdot, \lambda)\right)(t) d t\right) \\
= & i \lambda \theta_{1}(\tau, \lambda)+i v(\tau) \frac{1}{\sqrt{2}} e^{-i \tau \lambda} \\
& \times\left(-2 I_{r}+I_{r}+\int_{0}^{\tau}\left(\Gamma^{-1} k\right)(t)^{*}\left(\Gamma^{-1} \ell_{j}(\cdot, \lambda)\right)(t) d t\right) \\
= & i \lambda \theta_{1}(\tau, \lambda)+i v(\tau) \omega_{1}(\tau, \lambda),
\end{aligned}
$$

and

$$
\begin{aligned}
\frac{d}{d \tau} \theta_{2}(\tau, \lambda)= & -i \lambda \theta_{2}(\tau, \lambda)+2 i \lambda \theta_{2}(\tau, \lambda) \\
& +i v(\tau) \frac{1}{\sqrt{2}} e^{-i \tau \lambda}\left(I_{r}+\int_{0}^{\tau}\left(\Gamma^{-1} k\right)(t)^{*}\left(\Gamma^{-1} \ell_{2}(\cdot, \lambda)\right)(t) d t\right) \\
= & i \lambda \theta_{2}(\tau, \lambda)+i v(\tau) \omega_{2}(\tau, \lambda),
\end{aligned}
$$

Thus (2.23) is proved.

\section{Uniqueness of the Accelerant}

Let $u(x, \lambda)$ be the fundamental solution of the canonical system (1.10) satisfying the initial condition (2.2), and put

$$
\theta(x)=\left[\begin{array}{ll}
I_{r} & 0
\end{array}\right] u(x, 0), \quad 0 \leq x \leq \mathbf{T} .
$$

Since the potential $v$ of (1.10) is assumed to be continuous, the function $\theta$ is continuously differentiable on $[0, \mathbf{T}]$. With $\theta$ we associate a lower triangular semi-separable integral operator $L$ acting on $L_{r}^{2}(0, \mathbf{T})$, namely

$$
\begin{aligned}
(L f)(x) & =\theta(x) J \int_{0}^{x} \theta(t)^{*} f(t) d t, \quad 0 \leq x \leq \mathbf{T}, \\
J & =\left[\begin{array}{cc}
0 & I_{r} \\
I_{r} & 0
\end{array}\right] .
\end{aligned}
$$

Note that $L$ depends only on (1.10); accelerants do not play a role yet.

The operator $L$ will play an important role in the proof of the uniqueness of the accelerant (in the present section), and also later on in the construction of the accelerant given a continuous potential (in Sect. 5).

In this section $k$ is an accelerant for the canonical system (1.10), and we will show that $k$ is uniquely determined by the potential $v$. First we recall that the statement " $T$ is a convolution operator with kernel function $k$ " can 
be expressed in terms of an intertwining relation involving the operator of integration which is the operator $A$ on the space $L_{r}^{2}(0, \mathbf{T})$ defined by

$$
(A f)(x)=\int_{0}^{x} f(t) d t \quad(0 \leq x \leq \mathbf{T}) .
$$

In fact, using Theorem 1.2 in Chapter 1 of [14], we know that

$$
\begin{aligned}
& A T+T A^{*}=B J B^{*}, \text { where } J \text { is defined by }(3.3), \\
& B: \mathbb{C}^{2 r} \rightarrow L_{r}^{2}(0, \mathbf{T}), \quad B y=\frac{1}{\sqrt{2}} \ell(\cdot) y \quad\left(y \in \mathbb{C}^{2 r}\right) .
\end{aligned}
$$

Here $\ell$ is the $r \times 2 r$ matrix function given by

$$
\ell(x)=\left[\begin{array}{ll}
h(x) & I_{r}
\end{array}\right] \text { with } h(x)=I_{r}-2 \int_{0}^{x} k(t) d t, \quad 0 \leq x \leq \mathbf{T} .
$$

We shall need the following proposition and an additional lemma.

Proposition 3.1. Let $k$ be an accelerant for the canonical system (1.10), and let $T=\Gamma \Gamma^{*}$ be the $L U$-factrization of the corresponding convolution integral operator $T$. Then the operator $L$ defined by (3.2) is similar to the operator of integration $A$; in fact, $L=\Gamma^{-1} A \Gamma$.

Proof. We first show that $L+L^{*}=\Gamma^{-1} B J B^{*} \Gamma^{-*}$. The fact that $k$ is an accelerant for the canonical system (1.10) allows us to use the results of the previous section. Let $\ell_{1}(x, \lambda)$ and $\ell_{2}(x, \lambda)$ be the $r \times r$ matrix functions defined by (2.3). Note that $\ell_{1}(x, 0)$ is equal to $h(x)$, where $h$ is the function appearing in (3.7), and $\ell_{2}(x, 0)=I_{r}$. It follows that

$$
\ell(x)=\left[\ell_{1}(x, 0) \quad \ell_{2}(x, 0)\right], \quad 0 \leq x \leq \mathbf{T} .
$$

But then we see from (3.1), (2.7), and (2.20) that

$$
\theta(x)=\frac{1}{\sqrt{2}}\left(\Gamma^{-1} \ell\right)(x), \quad 0 \leq x \leq \mathbf{T} .
$$

Using the definition of $B$ in (3.6), the preceding identity yields $\Gamma^{-1} B y=\theta(\cdot) y$ for each $y \in \mathbb{C}^{2 r}$. As $L$ is defined by (3.2), we obtain $L+L^{*}=\Gamma^{-1} B J B^{*} \Gamma^{-*}$.

Next, since $T=\Gamma \Gamma^{*}$, the identity in (3.5) can be rewritten as

$$
A \Gamma \Gamma^{*}+\Gamma \Gamma^{*} A^{*}=B J B^{*} .
$$

Multiplying the latter identity from the left by $\Gamma^{-1}$ and from the right by the operator $\Gamma^{-*}$ yields

$$
\Gamma^{-1} A \Gamma+\left(\Gamma^{-1} A \Gamma\right)^{*}=\Gamma^{-1} B J B^{*} \Gamma^{-*} .
$$

By the result of the first paragraph, $L+L^{*}=\Gamma^{-1} B J B^{*} \Gamma^{-*}$. It follows that

$$
L-\Gamma^{-1} A \Gamma=\left(\Gamma^{-1} A \Gamma\right)^{*}-L^{*} .
$$

Note that the operator in the left hand side of (3.9) is a lower triangular integral operator of the first kind, while the operator in the right hand side of (3.9) is an upper triangular operator of the first kind. Hence both sides are equal to the zero operator. Thus $L=\Gamma^{-1} A \Gamma$. 
Lemma 3.2. Let $R$ be an operator on $L_{r}^{2}(0, \mathbf{T})$ commuting with the operator of integration $A$ given by (3.4). If, in addition, $(R u)(x)=u$ for each $u \in \mathbb{C}^{r}$ and each $0 \leq x \leq \mathbf{T}$, then $R$ is the identity operator on $L_{r}^{2}(0, \mathbf{T})$.

Proof. Let $\beta$ be the canonical embedding operator from $\mathbb{C}^{r}$ into $L_{r}^{2}(0, \mathbf{T})$, that is, $\beta$ is given by $(\beta u)(x)=u$ for each $u \in \mathbb{C}^{r}$ and $0 \leq x \leq \mathbf{T}$. Then $R \beta=\beta$. Since $R$ commutes with operator of integration $A$, we have $R A^{n} \beta=$ $A^{n} R \beta=A^{n} \beta$. Thus $R$ acts as the identity operator on the closed linear hull $\bigvee_{n=0}^{\infty} \operatorname{Im} A^{n} \beta$. By induction one shows that $\operatorname{Im} \beta+\operatorname{Im} A \beta+\cdots+\operatorname{Im} A^{n} \beta$ consists of all $\mathbb{C}^{r}$-valued polynomials of degree at most $n$. Since the set of all $\mathbb{C}^{r}$-valued polynomials is dense in $L_{r}^{2}(0, \mathbf{T})$, we conclude that $\bigvee_{n=0}^{\infty} \operatorname{Im} A^{n} \beta$ coincides with $L_{r}^{2}(0, \mathbf{T})$, and hence $R$ is identity operator on $L_{r}^{2}(0, \mathbf{T})$.

Theorem 3.3. The accelerant is uniquely determined by the potential.

Proof. By specifying (2.8) for $\lambda=0$ we see (using (2.12)) that

$$
\frac{1}{2} \sqrt{2}\left(\Gamma^{-1} I_{r}\right)(x)=\theta_{2}(x, 0)=\left[\begin{array}{ll}
I_{r} & 0
\end{array}\right] u(x, 0)\left[\begin{array}{c}
0 \\
I_{r}
\end{array}\right], \quad 0 \leq x \leq \mathbf{T} .
$$

Thus $\Gamma^{-1} I_{r}$ depends on the potential $v$ only and not on the particular choice of the accelerant.

Now fix the potential $v$, and let $\tilde{k}$ be another accelerant determining $v$. Thus $\tilde{k}$ is a hermitian $r \times r$ matrix function on the interval $-\mathbf{T} \leq t \leq \mathbf{T}$, which is is continuous on the interval $-\mathbf{T} \leq t \leq \mathbf{T}$ with possibly a jump discontinuity at the origin. Furthermore, the convolution integral operator $\tilde{T}$ defined by

$$
(\tilde{T} f)(x)=f(x)-\int_{0}^{\mathbf{T}} \tilde{k}(x-s) f(s) d s, \quad 0 \leq x \leq \mathbf{T},
$$

is a strictly positive operator on $L_{r}^{2}(0, \mathbf{T})$. Let $\tilde{\Gamma} \tilde{\Gamma}^{*}$ be the $L U$-factorization of $\tilde{T}$. Then Proposition 3.1, together with the fact that $L$ depends on (1.10) only, shows that $\Gamma^{-1} A \Gamma=\tilde{\Gamma}^{-1} A \tilde{\Gamma}$. In other words, the operator $\tilde{\Gamma} \Gamma^{-1}$ commutes with the operator $A$. The result of the first paragraph of the proof yields $\Gamma^{-1} I_{r}=\tilde{\Gamma}^{-1} I_{r}$. Thus the operator $\tilde{\Gamma} \Gamma^{-1}$ commutes with $A$ and $\left(\tilde{\Gamma} \Gamma^{-1} u\right)(x)=$ $u$ for each $u \in \mathbb{C}^{r}$ and $0 \leq x \leq \mathbf{T}$. According to Lemma 3.2 this implies that $\tilde{\Gamma} \Gamma^{-1}$ is the identity operator on $L_{r}^{2}(0, \mathbf{T})$. Hence $\tilde{\Gamma}=\Gamma$. But then $\tilde{T}=T$, and thus $\tilde{k}=k$. This proves the uniqueness of the accelerant.

\section{Semi-Separable Triangular Operators Similar to the Operator of Integration}

Throughout this section $K$ is a semi-separable lower triangular integral operator on $L_{r}^{2}(0, \mathbf{T})$, that is, the action of $K$ is given by

$$
(K f)(x)=F(x) \int_{0}^{x} G(t) f(t) d t, \quad f \in L_{r}^{2}(0, \mathbf{T}) .
$$


Here $F(\cdot)$ and $G(\cdot)$ are matrix functions of sizes $r \times p$ and $p \times r$, respectively, and their entries are square summable on the interval $[0, \mathbf{T}]$. In fact, we shall assume that $F(\cdot)$ and $G(\cdot)$ are continuously differentiable on $[0, \mathbf{T}]$ and such that

$$
F(x) G(x)=I_{r}, \quad 0 \leq x \leq \mathbf{T} .
$$

A simple example of such an operator is the operator of integration A on $L_{r}^{2}(0, \mathbf{T})$ defined by $(3.4)$.

We shall see that any semi-separable lower triangular operator $K$ satisfying the conditions referred to above is similar to the operator of integration $A$ and with a similarity operator of a special kind. The precise result is presented in the next proposition.

Proposition 4.1. Let $F$ and $G$ be continuously differentiable, and assume (4.2) holds. Then the operator $K$ defined by (4.1) is similar to the operator of integration A. More precisely, $K=E A E^{-1}$ where $E$ is a lower triangular operator of the form

$$
(E f)(x)=\rho(x) f(x)+\int_{0}^{x} e(x, t) f(t) d t, \quad f \in L_{r}^{2}(0, \mathbf{T}) .
$$

Here, $e(x, t)$ is a continuous $r \times r$ matrix function on $0 \leq t \leq x \leq \mathbf{T}$, which is zero at $t=0$, and the $r \times r$ matrix function $\rho$ is given by

$$
\frac{d}{d x} \rho(x)=F^{\prime}(x) G(x) \rho(x), \quad \rho(0)=I_{r} .
$$

Moreover, the operators $E^{ \pm 1}$ map functions with a continuous derivative into functions with a continuous derivative.

When $F$ and $G$ are boundedly differentiable and continuous derivatives are replaced by bounded derivatives, the above proposition is a particular case of Theorem 1 in [11]. The restriction to continuously differentiable $F$ and $G$ is the new element here. Since the above proposition plays an essential role in the proof of our main theorem, we will present a full proof.

In order to prove Proposition 4.1 we first make some heuristic remarks explaining the line of reasoning that we will follow. Assume we have an operator $E$ on $L_{r}^{2}(0, \mathbf{T})$ with all the properties described in Proposition 4.1. In particular, $K E=E A$. By rewriting this identity in terms of the kernel functions of the integral operators $A, K, E$, we get

$$
F(x) G(t) \rho(t)+F(x) \int_{t}^{x} G(s) e(s, t) d s=\rho(x)+\int_{t}^{x} e(x, s) d s .
$$

Taking $t=0$ and using $e(x, 0)=0$ for each $0 \leq x \leq \mathbf{T}$, we conclude that

$$
\left(E I_{r}\right)(x)=\rho(x)+\int_{0}^{x} e(x, s) d s=F(x) G(0), \quad 0 \leq x \leq \mathbf{T} .
$$

Here we view $I_{r}$ as the $r \times r$ matrix function on $[0, \mathbf{T}]$ which is identically equal to the $r \times r$ identity matrix, and $E$ is applied to $I_{r}$ column wise. 
On the other hand, since $K$ and $A$ are Volterra operators (cf., Section 12.9 in [2]) the identity $K E=E A$ implies that $(I-\lambda K)^{-1} E=E(I-\lambda A)^{-1}$ for each $\lambda \in \mathbb{C}$. Here, and in the sequel, $I$ denotes the identity operator on $L_{r}^{2}(0, \mathbf{T})$. As is well known, for each $f$ in $L_{r}^{2}(0, \mathbf{T})$ we have

$$
\left((I-\lambda A)^{-1} f\right)(x)=f(x)+\lambda \int_{0}^{x} e^{\lambda(x-t)} f(t) d t, \quad 0 \leq x \leq \mathbf{T} .
$$

With $f(\cdot)=I_{r}$ this yields

$$
\left((I-\lambda A)^{-1} I_{r}\right)(x)=e^{\lambda x} I_{r}, \quad 0 \leq x \leq \mathbf{T} .
$$

It follows (using the identity (4.5)) that

$$
\begin{aligned}
& \rho(x) e^{\lambda x} I_{r}+\int_{0}^{x} e(x, t) e^{\lambda t} I_{r} d t \\
& \quad=\left(E\left(e^{\lambda \cdot} I_{r}\right)\right)(x)=\left(E(I-\lambda A)^{-1} I_{r}\right)(x) \\
& \quad=\left((I-\lambda K)^{-1} E I_{r}\right)(x)=\left((I-\lambda K)^{-1} F(\cdot) G(0)\right)(x), \quad 0 \leq x \leq \mathbf{T} .
\end{aligned}
$$

Hence in order to find the kernel function $e(x, t)$ it is natural to solve the equation $(I-\lambda K) g(\cdot, \lambda)=F(\cdot) G(0)$ and to analyze its solution. This will be done in Lemmas 4.2 and 4.3 below.

We begin with some preparations According to the general theory of semiseparable integral operators (see Chapter IX in [3]), the inverse of operator $I-\lambda K$ is given by

$$
\left((I-\lambda K)^{-1} f\right)(x)=f(x)+\int_{0}^{x} \eta(x, t, \lambda) f(t) d t,
$$

where and

$$
\begin{aligned}
\eta(x, t, \lambda) & =\lambda F(x) u_{1}(x, \lambda) u_{1}(t, \lambda)^{-1} G(t), \quad 0 \leq t \leq x \leq \mathbf{T} \\
\frac{d}{d x} u_{1}(x, \lambda) & =\lambda G(x) F(x) u_{1}(x, \lambda), \quad 0 \leq x \leq \mathbf{T} \\
u_{1}(0, \lambda) & =I_{r}
\end{aligned}
$$

We also need the $r \times r$ matrix function $\widetilde{u}_{1}(x)$ defined by

$$
\frac{d}{d x} \widetilde{u}_{1}(x)=-G(x) F^{\prime}(x) \widetilde{u}_{1}(x), \quad 0 \leq x \leq \mathbf{T}, \quad \widetilde{u}_{1}(0)=I_{r} .
$$

We are now ready to prove the first lemma.

Lemma 4.2. Let $F$ and $G$ be continuously differentiable, and assume (4.2) holds. Let $h$ be the $r \times r$ matrix function defined by $h(x)=F(x) G(0)$ on $0 \leq x \leq \mathbf{T}$, and let $\rho$ be the $r \times r$ matrix function given by (4.4). Put

$$
g(x, \lambda)=\rho(x)^{-1}\left((I-\lambda K)^{-1} h\right)(x), \quad 0 \leq x \leq \mathbf{T},
$$


where $(I-\lambda K)^{-1}$ is applied to $h$ columnwise. Then $g$ satisfies the following integro-differential equation

$$
\frac{d}{d x} g(x, \lambda)-\alpha(x) \int_{0}^{x} \beta(t) g(t, \lambda) d t-\lambda g(x, \lambda)=0, \quad g(0, \lambda)=I_{r},
$$

where $\alpha$ and $\beta$ are the continuous functions on $[0, \mathbf{T}]$ given by

$$
\begin{aligned}
\alpha(x) & =\rho(x)^{-1} F^{\prime}(x) \widetilde{u}_{1}(x), \quad 0 \leq x \leq \mathbf{T}, \\
\beta(t) & =-\widetilde{u}_{1}(t)^{-1}\left(G(t) F^{\prime}(t) G(t)+G^{\prime}(t)\right) \rho(t), \quad 0 \leq t \leq \mathbf{T} .
\end{aligned}
$$

Proof. Put $\widetilde{g}(x, \lambda)=\rho(x) g(x, \lambda)$. Using (4.8)-(4.11), (4.13), and the definition of the matrix function $h$, we present $\widetilde{g}$ in the form

$$
\begin{aligned}
& \widetilde{g}(x, \lambda) \\
& \quad=F(x) G(0)+\lambda F(x) u_{1}(x, \lambda) \int_{0}^{x} u_{1}(t, \lambda)^{-1} G(t) F(t) G(0) d t \\
& \quad=F(x) G(0)-F(x) u_{1}(x, \lambda) \int_{0}^{x} \frac{d}{d t}\left(u_{1}(t, \lambda)^{-1} G(0)\right) d t \\
& \quad=F(x) G(0)-F(x) u_{1}(x, \lambda)\left(u_{1}(x, \lambda)^{-1}-I_{r}\right) G(0) \\
& \quad=F(x) u_{1}(x, \lambda) G(0) .
\end{aligned}
$$

It follows that

$$
g(x, \lambda)=\rho(x)^{-1} F(x) u_{1}(x, \lambda) G(0) .
$$

Clearly $g$ is differentiable and

$$
\begin{aligned}
\frac{d}{d x} g(x, \lambda) & =\rho(x)^{-1} \widetilde{g}_{x}(x, \lambda)-\rho(x)^{-1} \rho^{\prime}(x) \rho(x)^{-1} \widetilde{g}(x, \lambda) \\
& =\rho(x)^{-1}\left\{\lambda F(x) G(x) F(x)+F^{\prime}(x)-F^{\prime}(x) G(x) F(x)\right\} u_{1}(x, \lambda) G(0) \\
& =\lambda g(x, \lambda)+\rho(x)^{-1} F^{\prime}(x)\left(I_{p}-G(x) F(x)\right) u_{1}(x, \lambda) G(0) .
\end{aligned}
$$

Here we took into account the identity (4.2). From (4.12) we see that

$$
\frac{d}{d t} \widetilde{u}_{1}(t)^{-1}=-\widetilde{u}_{1}(t)^{-1}\left(\frac{d}{d t} \widetilde{u}_{1}(t)\right) \widetilde{u}_{1}(t)^{-1}=\widetilde{u}_{1}(t)^{-1} G(t) F^{\prime}(t) .
$$

Hence

$$
\begin{aligned}
\frac{d}{d t} & \left(\widetilde{u}_{1}(t)^{-1}\left(I_{p}-G(t) F(t)\right) u_{1}(t, \lambda)\right) \\
= & \widetilde{u}_{1}(t)^{-1} G(t) F^{\prime}(t)\left(I_{p}-G(t) F(t)\right) u_{1}(t, \lambda) \\
& +\widetilde{u}_{1}(t)^{-1}\left(-G^{\prime}(t) F(t)-G(t) F^{\prime}(t)\right) u_{1}(t, \lambda) \\
& +\lambda \widetilde{u}_{1}(t)^{-1}\left(I_{p}-G(t) F(t)\right) G(t) F(t) u_{1}(t, \lambda) .
\end{aligned}
$$


Since $\left(I_{p}-G(t) F(t)\right) G(t)=0$ because of condition (4.2), we see that

$$
\begin{aligned}
\frac{d}{d t}( & \left.\widetilde{u}_{1}(t)^{-1}\left(I_{p}-G(t) F(t)\right) u_{1}(t, \lambda)\right) \\
= & \widetilde{u}_{1}(t)^{-1}\left(G(t) F^{\prime}(t)-G(t) F^{\prime}(t) G(t) F(t)\right. \\
& \left.-G^{\prime}(t) F(t)-G(t) F^{\prime}(t)\right) u_{1}(t, \lambda) \\
= & -\widetilde{u}_{1}(t)^{-1}\left(G(t) F^{\prime}(t) G(t)+G^{\prime}(t)\right) F(t) u_{1}(t, \lambda) .
\end{aligned}
$$

Using the definition of $\beta$ in (4.16) and the identity (4.18), we obtain

$$
\frac{d}{d t}\left(\widetilde{u}_{1}(t)^{-1}\left(I_{p}-G(t) F(t)\right) u_{1}(t, \lambda)\right) G(0)=\beta(t) g(t, \lambda) .
$$

Recall that $\left(I_{p}-G(t) F(t)\right) G(t)=0$ and $\left(I_{p}-G(0) F(0)\right) G(0)=0$, in particular. From integration by parts it follows that

$$
\begin{aligned}
\int_{0}^{x} \beta(t) g(t, \lambda) d t= & \widetilde{u}_{1}(x)^{-1}\left(I_{p}-G(x) F(x)\right) u_{1}(x, \lambda) G(0) \\
& -\left(I_{p}-G(0) F(0)\right) G(0)-\lambda \int_{0}^{x} \widetilde{u}_{1}(t)^{-1}\left(I_{p}-G(t) F(t)\right) G(t) \\
& \times F(t) u_{1}(t, \lambda) G(0) d t \\
= & \widetilde{u}_{1}(x)^{-1}\left(I_{p}-G(x) F(x)\right) u_{1}(x, \lambda) G(0) .
\end{aligned}
$$

But then, using (4.19) and the definition of $\alpha$ in (4.15), we arrive at the identity (4.14).

The following lemma provides an integral representation of $g$.

Lemma 4.3. Let $\gamma(x, t)$ be an $r \times r$ matrix function, continuous on the interval $0 \leq t \leq x \leq \mathbf{T}$. Then the integro-differential equation

$$
\frac{d}{d x} g(x, \lambda)-\int_{0}^{x} \gamma(x, t) g(t, \lambda) d t-\lambda g(x, \lambda)=0, \quad g(0, \lambda)=I_{r},
$$

has a unique continuously differentiable solution $g$. Moreover, $g$ is of the form

$$
g(x, \lambda)=e^{\lambda x} I_{r}+\int_{0}^{x} e^{\lambda t} N(x, t) d t, \quad 0 \leq x \leq \mathbf{T},
$$

where $N(x, t)$ is continuous on $0 \leq t \leq x \leq \mathbf{T}$ and $N(x, 0)=0$ for each $0 \leq x \leq \mathbf{T}$.

Proof. Throughout the proof we fix $\lambda \in \mathbb{C}$. By definition a solution $g(x, \lambda)$ of (4.21) is absolutely continuous on $0 \leq x \leq \mathbf{T}$. In that case, since $\gamma(x, t)$ is continuous on $0 \leq t \leq x \leq \mathbf{T}$, we see that

$$
\lambda g(x, \lambda)+\int_{0}^{x} \gamma(x, t) g(t, \lambda) d s \text { is continuous on } 0 \leq x \leq \mathbf{T} .
$$


But then $\frac{d}{d x} g(x, \lambda)$ is also continuous on $0 \leq x \leq \mathbf{T}$. Thus any solution of (4.21) is automatically continuously differentiable.

By integrating the equation in (4.21) over $0 \leq x \leq \tau$, where $0 \leq \tau \leq \mathbf{T}$, we obtain the equation

$$
g(\cdot, \lambda)-\lambda A g(\cdot, \lambda)-A R g(\cdot, \lambda)=I_{r}
$$

Here $A$ is the operator of integration on $L_{r}^{2}(0, \mathbf{T})$ defined by $(3.4)$ and $R$ is the operator on $L_{r}^{2}(0, \mathbf{T})$ given by

$$
(R f)(x)=\int_{0}^{x} \gamma(x, t) f(t) d t, \quad f \in L_{r}^{2}(0, \mathbf{T})
$$

Note that for each $f$ in $L_{r}^{2}(0, \mathbf{T})$ the function $A f$ is absolutely continuous on $[0, \mathbf{T}]$. It follows that any solution of (4.23) is absolutely continuous, and thus the problems (4.21) and (4.23) are equivalent. Using (4.6) we get

$$
\left((I-\lambda A)^{-1} A f\right)(x)=\int_{0}^{x} e^{\lambda(x-t)} f(t) d t, \quad 0 \leq x \leq \mathbf{T} .
$$

From (4.24) and the definition of $R$ we see that for each $f$ in $L_{r}^{2}(0, \mathbf{T})$

$$
\left((I-\lambda A)^{-1} A R f\right)(x)=\int_{0}^{x} \widetilde{\gamma}(x, t ; \lambda) f(t) d t, \quad 0 \leq x \leq \mathbf{T},
$$

where

$$
\widetilde{\gamma}(x, t ; \lambda)=\int_{t}^{x} e^{\lambda(x-s)} \gamma(s, t) d s, \quad 0 \leq t \leq x \leq \mathbf{T} .
$$

It follows that $I-(I-\lambda A)^{-1} A R$ is an invertible operator on $L_{r}^{2}(0, \mathbf{T})$. Hence the problem (4.23) has a unique solution in $L_{r}^{2}(0, \mathbf{T})$, namely

$$
g(\cdot, \lambda)=\left(I-(I-\lambda A)^{-1} A R\right)^{-1}(I-\lambda A)^{-1} I_{r}
$$

We conclude that Eq. (4.21) has a unique continuously differentiable solution.

It remains to show that the solution $g(\cdot, \lambda)$ is of the form $(4.22)$. To do this, we use (4.7) and rewrite (4.25) as

$$
g(\cdot, \lambda)=e^{\lambda \cdot} I_{r}+\sum_{k=1}^{\infty}\left((I-\lambda A)^{-1} A R\right)^{k} e^{\lambda \cdot} I_{r}
$$


Let us compute the first term with $k=1$. Using (4.24) and the definition of $R$, we get

$$
\begin{aligned}
\left(\left((I-\lambda A)^{-1} A R\right) e^{\lambda \cdot} I_{r}\right)(x) & =\int_{0}^{x} e^{\lambda(x-r)}\left(\int_{0}^{r} \gamma(r, t) e^{\lambda t} I_{r} d t\right) d r \\
& =\int_{0}^{x}\left(\int_{0}^{r} e^{\lambda(x+t-r)} \gamma(r, t) d t\right) d r \\
& =\int_{0}^{x}\left(\int_{x-r}^{x} e^{\lambda t} \gamma(r, r+t-x) d t\right) d r \\
& =\int_{0}^{x} e^{\lambda t}\left(\int_{x-t}^{x} \gamma(r, r+t-x) d r\right) d t \\
& =\int_{0}^{x} e^{\lambda t} \gamma_{1}(x, t) d t,
\end{aligned}
$$

where

$$
\gamma_{1}(x, t)=\int_{x-t}^{x} \gamma(r, r+t-x) d r, \quad 0 \leq t \leq x \leq \mathbf{T} .
$$

Next define matrix $\gamma_{k}(t, s), k=2,3, \ldots$, recursively by

$$
\gamma_{k+1}(x, t)=\int_{x-t}^{x} \int_{y+t-x}^{y} \gamma(y, s) \gamma_{k}(s, t+y-x) d s d y .
$$

Then, using similar calculations as for $k=1$ above, one proves by induction that for each $k \geq 1$ we have

$$
\left(\left((I-\lambda A)^{-1} A R\right)^{k} e^{\lambda \cdot} I_{r}\right)(x)=\int_{0}^{x} e^{\lambda t} \gamma_{k}(x, t) d t, \quad 0 \leq x \leq \mathbf{T} .
$$

Observe that for each $k$ the function $\gamma_{k}(x, t)$ is continuous on $0 \leq t \leq x \leq \mathbf{T}$. Furthermore, as we see from (4.27) and (4.28), we have

$$
\left\|\gamma_{k}(x, t)\right\| \leq c^{k} \frac{x^{2 k-1}}{(2 k-1) !}, \quad 0 \leq t \leq x \leq \mathbf{T}, \quad k \geq 1 .
$$

Here $c$ is a constant independent of $k$. Finally, using (4.26), we conclude that (4.22) holds with

$$
N(x, t)=\sum_{k=1}^{\infty} \gamma_{k}(x, t), \quad 0 \leq t \leq x \leq \mathbf{T} .
$$

By (4.30) the convergence in the preceding formula is uniform on the triangle $0 \leq t \leq x \leq \mathbf{T}$. Since each of the terms $\gamma_{k}(x, t)$ is continuous on this triangle, it follows that $N(x, t)$ is continuous on $0 \leq t \leq x \leq \mathbf{T}$ as desired. Finally, 
from (4.27) and (4.28) it is clear that $\gamma_{k}(x, 0)=0$ for each $0 \leq x \leq \mathbf{T}$ and each positive integer $k$. But then $N(\cdot, 0)$ is identically equal to zero too.

Proof of Proposition 4.1. We split the proof into three parts. In the first part we define the operator $E$ and establish the similarity $K E=E A$. In the two other parts we prove that $E^{ \pm 1}$ map functions with a continuous derivative into functions with a continuous derivative.

Part 1. Let $g(x, \lambda)$ be the matrix function defined by (4.13). From Lemma 4.2 we know that $g(x, \lambda)$ satisfies the integro-differential equation (4.14). But then we can apply Lemma 4.3 with $\gamma(x, t)=\alpha(x) \beta(t)$, where $\alpha(\cdot)$ and $\beta(\cdot)$ are defined by (4.15) and (4.16). It follows that $g$ admits the representation

$$
g(x, \lambda)=e^{\lambda x} I_{r}+\int_{0}^{x} N(x, t) e^{\lambda t} I_{r} d t, \quad 0 \leq x \leq \mathbf{T},
$$

with $N(x, t)$ being continuous on $0 \leq t \leq x \leq \mathbf{T}$ and with $N(\cdot, 0)$ identically equal to zero. Now let $E$ be the operator on $L_{r}^{2}(0, \mathbf{T})$ defined by

$$
(E f)(x)=\rho(x) f(x)+\int_{0}^{x} \rho(x) N(x, t) f(t) d t, \quad 0 \leq x \leq \mathbf{T} .
$$

Here the $r \times r$ matrix function $\rho$ is defined by (4.4). Thus $E$ has the form (4.3) with $e(x, t)=\rho(x) N(x, t)$. Obviously $e(x, t)$ is continuous on $0 \leq t \leq x \leq \mathbf{T}$ and $e(\cdot, 0)=0$ on $[0, \mathbf{T}]$. We claim that this operator $E$ has all the properties described in Proposition 4.1.

From (4.7), (4.13), formula (4.32) applied to $f=e^{\lambda \cdot} I_{r}$, and the identity in $(4.31)$ we see that

$$
E(I-\lambda A)^{-1} I_{r}=E\left(e^{\lambda \cdot} I_{r}\right)=\rho(\cdot) g(\cdot, \lambda)=(I-\lambda K)^{-1} h,
$$

where $h=F(\cdot) G(0)$. Taking $\lambda=0$ in the above identity, we obtain $h=E I_{r}$. Therefore,

$$
(I-\lambda K)^{-1} E I_{r}=E(I-\lambda A)^{-1} I_{r} .
$$

From the series expansion in (4.33) it follows that

$$
K^{j} E I_{r}=E A^{j} I_{r}, \quad j=0,1,2, \ldots
$$

Therefore, for each $j=0,1,2, \ldots$, we have

$$
(K E) A^{j} I_{r}=K\left(E A^{j} I_{r}\right)=K^{j+1} E I_{r}=E A^{j+1} I_{r}=(E A) A^{j} I_{r} .
$$

As the closed linear span of the columns of the matrices $\left\{A^{j} I_{r}\right\}_{j=0}^{\infty}$ coincides with $L_{r}^{2}(0, \mathbf{T})$, the equalities in (4.35) yield $K E=E A$. Since $E$ is invertible, we obtain $K=E A E^{-1}$, and hence $K$ and $A$ are similar. It remains to prove that $E^{ \pm 1}$ map functions with a continuous derivative into functions with a continuous derivative.

Part 2. To show that $E$ has this property, let $f$ be any $\mathbb{C}^{n}$-valued function on $[0, \mathbf{T}]$ with a continuous derivative. Then $f(\cdot)=(A g)(\cdot)+u$, where $g$ is the derivative of $f$ and $u$ is a constant $r \times r$ matrix. As we have seen in the previous paragraph, $E I_{r}=h=F(\cdot) G(0)$. Thus $E u=F(\cdot) G(0) u$. According 
to our hypotheses, $F(\cdot)$ is continuously differentiable. Hence the same holds true for $E u$. Next note that

$$
(E A g)(x)=(K E g)(x)=F(x) \int_{0}^{x} G(t)(E g)(t) d t \text {. }
$$

Since $\rho$ is continuous on $[0, \mathbf{T}]$ and $e(x, t)$ is continuous on $0 \leq t \leq x \leq \mathbf{T}$, we know that $E$ maps continuous functions into continuous functions. In particular, $E g$ is continuous, and hence the above formula shows that $E A g$ has a continuous derivative. Therefore, $E f=E A g+E u$ is continuously differentiable.

Part 3. Next, we prove that $E^{-1}$ maps functions with a continuous derivative into functions with a continuous derivative. First notice that $E^{-1}$ admits the representation

$$
\left(E^{-1} f\right)(x)=\rho(x)^{-1} f(x)+\int_{0}^{x} e^{\times}(x, t) f(t) d t, \quad f \in L_{r}^{2}(0, \mathbf{T}) .
$$

As $e(x, t)$ is continuous on $0 \leq t \leq x \leq \mathbf{T}$, the same holds true for $e^{\times}(x, t)$, and thus $E^{-1}$ maps continuous function into continuous functions. In terms of the kernel functions the identity $E^{-1} E=I$ means

$$
e^{\times}(x, t) \rho(t)+\rho(x)^{-1} e(x, t)+\int_{t}^{x} e^{\times}(x, s) e(s, t) d s=0, \quad 0 \leq t \leq x \leq \mathbf{T} .
$$

Recall that $e(\cdot, 0) \equiv 0$. Thus by taking $t=0$ in the preceding identity we obtain $e^{\times}(x, 0)=0$ for $0 \leq x \leq \mathbf{T}$.

We shall need the operator $K_{1}$ on $L_{r}^{2}(0, \mathbf{T})$ defined by

$$
\left(K_{1} f\right)(x)=F^{\prime}(x) \int_{0}^{x} G(t) d t, \quad f \in L_{r}^{2}(0, \mathbf{T}) .
$$

Here $F^{\prime}$ is the derivative of $F$, which is a continuous function on $[0, \mathbf{T}]$. Notice that $K=A\left(I+K_{1}\right)$. Since $K E=E A$, we have $E^{-1} K=A E^{-1}$ which yields

$$
\begin{aligned}
E^{-1} A & =E^{-1} A\left(I+K_{1}\right)\left(I+K_{1}\right)^{-1}=E^{-1} K\left(I+K_{1}\right)^{-1} \\
& =A E^{-1}\left(I+K_{1}\right)^{-1} .
\end{aligned}
$$

Since the kernel function $F^{\prime}(x) G(t)$ of $K_{1}$ is continuous, $\left(I+K_{1}\right)^{-1}$ maps continuous functions into continuous functions.

Now let $f$ be a $\mathbb{C}^{r}$-valued function on $[0, \mathbf{T}]$ with a continuous derivative. As in the previous part, we can represent $f$ as $f(\cdot)=(A g)(\cdot)+u$, where $g$ is the derivative of $f$ and $u$ is a constant $r \times r$ matrix. According to (4.37) we have $E^{-1} A g=A E^{-1}\left(I+K_{1}\right)^{-1} g$. Since both $E^{-1}$ and $\left(I+K_{1}\right)^{-1}$ map continuous functions into continuous functions, the function $E^{-1}\left(I+K_{1}\right)^{-1} g$ is continuous. Thus $E^{-1} A g$ has a continuous derivative.

Hence in order to prove that $E^{-1} f$ has a continuous derivative, it suffices to show that $E^{-1} I_{r}$ has a continuous derivative. By rewriting the identity 
$E^{-1} K=A E^{-1}$ in terms of the kernel functions of $A, K$, and $E^{-1}$ we get

$$
\rho(x)^{-1} F(x) G(t)+\int_{t}^{x} e^{\times}(x, s) F(s) d s G(t)=\rho(t)^{-1}+\int_{t}^{x} e^{\times}(s, t) d s .
$$

By taking $t=0$ and using $e^{\times}(x, 0)=0$ for $0 \leq x \leq \mathbf{T}$ we obtain

$$
I_{r}-\rho(x)^{-1} F(x) G(0)-\int_{0}^{x} e^{\times}(x, s) F(s) d s G(0)=0 .
$$

Since $F(0) G(0)=I_{r}$, we can use (4.36) and (4.38) to show that

$$
\begin{aligned}
\left(E^{-1} I_{r}\right)(x)= & I_{r}-\rho(x)^{-1}(F(x)-F(0)) G(0) \\
& -\int_{0}^{x} e^{\times}(x, s)(F(s)-F(0)) d s G(0) .
\end{aligned}
$$

Using $\left(A F^{\prime}(\cdot) G(0)\right)(x)=((F(x)-F(0)) G(0)$, it follows from (4.39) and (4.36) that $\left(E^{-1} I_{r}\right)(x)=I_{r}-\left(E^{-1} A F^{\prime}(\cdot)\right)(x) G(0)$. As the right-hand side of the latter identity has a continuous derivative, we obtain that $E^{-1} I_{r}$ is continuously differentiable.

\section{Construction of an Accelerant}

In this section we establish the main part of Theorem 1.2. Throughout the $2 r \times 2 r$ matrix function $u(x, \lambda)$ is the fundamental solution of the canonical system (1.10) normalized by

$$
u(0, \lambda)=Q^{*}, \quad \text { where } Q=\frac{1}{\sqrt{2}}\left[\begin{array}{ll}
I_{r} & -I_{r} \\
I_{r} & I_{r}
\end{array}\right] .
$$

The potential $v$ of (1.10) is assumed to be continuous on [0, T]. Finally, $j$ and $J$ are signature matrices, $j$ is defined by (1.11) and $J$ by (3.3). Our aim is to show that $v$ is generated by an accelerant.

In what follows $\theta$ and $\omega$ are the $r \times 2 r$ matrix functions on $[0, \mathbf{T}]$ defined by

$$
\begin{aligned}
\theta(x) & =\left[\begin{array}{cc}
I_{r} & 0
\end{array}\right] u(x, 0), \quad 0 \leq x \leq \mathbf{T}, \\
\omega(x) & =\left[\begin{array}{ll}
0 & I_{r}
\end{array}\right] u(x, 0), \quad 0 \leq x \leq \mathbf{T} .
\end{aligned}
$$

We begin with two lemmas. The first will enable us to use Proposition 4.1.

Lemma 5.1. Let $\theta$ be the $r \times 2 r$ matrix function on $[0, \mathbf{T}]$ defined by (5.2). Then $\theta$ is continuously differentiable on $[0, \mathbf{T}]$,

$$
\theta(x) J \theta(x)^{*}=I_{r} \quad \text { and } \quad \theta^{\prime}(x) J \theta(x)^{*}=0 \quad(0 \leq x \leq \mathbf{T}) .
$$

Proof. It is straightforward to check that $Q$ defined in (5.1) satisfies the identities

$$
Q^{*}=Q^{-1}, \quad Q j Q^{*}=J, \quad Q^{*} J Q=j .
$$


Since $u(x, \lambda)$ satisfies (1.10) and the potential $v$ is continuous, the function $u(x, \lambda)$ is continuously differentiable in $x$. Furthermore, again using that $u(x, \lambda)$ satisfies $(1.10)$, we have $\frac{d}{d x}\left(u(x, \bar{\lambda})^{*} j u(x, \lambda)\right)=0$. Hence, taking into account (5.1) and (5.5), we derive

$$
u(x, \bar{\lambda})^{*} j u(x, \lambda)=J, \quad u(x, \lambda) J u(x, \bar{\lambda})^{*}=j .
$$

The fact that $u(x, \lambda)$ is continuously differentiable in $x$, implies that $\theta$ is continuously differentiable on $[0, \mathbf{T}]$. Furthermore, from (5.6) and (1.10) we see that for each $0 \leq x \leq \mathbf{T}$ we have

$$
\begin{aligned}
\theta(x) J \theta(x)^{*} & =\left[\begin{array}{ll}
I_{r} & 0
\end{array}\right] u(x, 0) J u(x, 0)^{*}\left[\begin{array}{c}
I_{r} \\
0
\end{array}\right]=I_{r}, \\
\theta^{\prime}(x) J \theta(x)^{*} & =\left[\begin{array}{ll}
I_{r} & 0
\end{array}\right]\left(\frac{d}{d x} u(x, 0)\right) J u(x, 0)^{*}\left[\begin{array}{c}
I_{r} \\
0
\end{array}\right]=0 .
\end{aligned}
$$

Thus the identities in (5.4) hold.

Lemma 5.2. Let $\omega$ be the $r \times 2 r$ matrix function defined by (5.3), and let $\theta$ be as in (5.2). Then $\omega$ is continuously differentiable on $[0, \mathbf{T}]$, and for each $0 \leq x \leq \mathbf{T}$ we have

$$
\theta(x) J \omega(x)^{*}=0, \quad \omega^{\prime}(x) J \omega(x)^{*}=0, \quad \omega(0)=\frac{1}{\sqrt{2}}\left[\begin{array}{ll}
-I_{r} & I r
\end{array}\right] .
$$

Moreover, the three identities in (5.9) determine $\omega$ uniquely. Finally,

$$
\omega^{\prime}(x) J \theta(x)^{*}=-i v(x)^{*}, \quad 0 \leq x \leq \mathbf{T} .
$$

Proof. Since $u(x, \lambda)$ is continuously differentiable in $x$ on $[0, \mathbf{T}]$, the same holds true for $\omega(x)$. From the second identity in (5.6) and the definitions of $\theta$ and $\omega$ in (5.2) and in (5.3), respectively, we get

$$
\theta(x) J \omega(x)^{*}=\left[\begin{array}{ll}
I_{r} & 0
\end{array}\right] u(x, 0) J u(x, 0)^{*}\left[\begin{array}{c}
0 \\
I_{r}
\end{array}\right]=\left[\begin{array}{ll}
I_{r} & 0
\end{array}\right] j\left[\begin{array}{c}
0 \\
I_{r}
\end{array}\right]=0 .
$$

Analogously, using (1.10),

$$
\begin{aligned}
\omega^{\prime}(x) J \omega(x)^{*} & =\left[\begin{array}{ll}
0 & I_{r}
\end{array}\right]\left(\frac{d}{d x} u(x, 0)\right) J u(x, 0)^{*}\left[\begin{array}{c}
0 \\
I_{r}
\end{array}\right] \\
& =\left[\begin{array}{ll}
0 & I_{r}
\end{array}\right]\left[\begin{array}{cc}
0 & i v(x) \\
-i v(x)^{*} & 0
\end{array}\right] j\left[\begin{array}{c}
0 \\
I_{r}
\end{array}\right]=0 .
\end{aligned}
$$

Thus the first two identities in (5.9) are proved. The third follows directly the normalizing condition (5.1).

To prove that the three identities in (5.9) determine $\omega$ uniquely, note that the second identity in (5.9) implies that

$$
\frac{d}{d x}\left(\omega(x) J \omega(x)^{*}\right)=\omega^{\prime}(x) J \omega(x)^{*}+\omega(x) J\left(\omega^{\prime}(x)\right)^{*}=0 .
$$


Thus, using the third identity in (5.9), we obtain $\omega(x) J \omega(x)^{*}=-I_{r}$. The latter identity, together with the first identity in (5.9), yields

$$
u(x, 0) J \omega(x)^{*}=\left[\begin{array}{c}
0 \\
-I_{r}
\end{array}\right] .
$$

Let $\tilde{\omega}$ be another continuously differentiable function on $[0, \mathbf{T}]$ such that the three identities in (5.9) hold with $\tilde{\omega}$ in place of $\omega$. Repeating the above reasoning for $\tilde{\omega}$ in place of $\omega$ we see that (5.11) holds for $\tilde{\omega}$ in place of $\omega$. Thus $u(x, 0) J\left(\omega(x)^{*}-\tilde{\omega}(x)^{*}\right)=0$. But the matrices $u(x, 0)$ and $J$ are nonsingular. Thus $\tilde{\omega}(x)=\omega(x)$ for each $x \in[0, \mathbf{T}]$. It follows that $\omega$ is uniquely determined by the identities in (5.9).

To prove the final identity (5.10) we use (1.10) and the definitions of $\theta$ and $\omega$ in (5.2) and (5.3). This yields

$$
\begin{aligned}
\omega^{\prime}(x) J \theta(x)^{*} & =\left[\begin{array}{ll}
0 & I_{r}
\end{array}\right]\left(\frac{d}{d x} u(x, 0)\right) J u(x, 0)^{*}\left[\begin{array}{c}
I_{r} \\
0
\end{array}\right] \\
& =\left[\begin{array}{ll}
0 & I_{r}
\end{array}\right]\left[\begin{array}{cc}
0 & i v(x) \\
-i v(x)^{*} & 0
\end{array}\right]\left[\begin{array}{c}
I_{r} \\
0
\end{array}\right]=-i v(x)^{*} .
\end{aligned}
$$

Hence (5.10) is proved.

In what follows it will be convenient to use the following notation:

$$
\begin{aligned}
& \theta_{0,1}(x)=\theta(x)\left[\begin{array}{c}
I_{r} \\
0
\end{array}\right], \quad \theta_{0,2}(x)=\theta(x)\left[\begin{array}{c}
0 \\
I_{r}
\end{array}\right] \quad(0 \leq x \leq \mathbf{T}) \\
& \omega_{0,1}(x)=\omega(x)\left[\begin{array}{c}
I_{r} \\
0
\end{array}\right], \quad \omega_{0,2}(x)=\omega(x)\left[\begin{array}{c}
0 \\
I_{r}
\end{array}\right] \quad(0 \leq x \leq \mathbf{T}) .
\end{aligned}
$$

Thus

$$
u(x, 0)=\left[\begin{array}{c}
\theta(x) \\
\omega(x)
\end{array}\right]=\left[\begin{array}{ll}
\theta_{0,1}(x) & \theta_{0,2}(x) \\
\omega_{0,1}(x) & \omega_{0,2}(x)
\end{array}\right], \quad 0 \leq x \leq \mathbf{T} .
$$

We now return to the operator $L$ defined by (3.2). Thus $L$ is the lower triangular semi-separable integral operator on $L_{r}^{2}(0, \mathbf{T})$ defined by

$$
(L f)(x)=\theta(x) J \int_{0}^{x} \theta(t)^{*} f(t) d t, \quad 0 \leq x \leq \mathbf{T} .
$$

Here $\theta$ is as in (5.2) (cf., (3.1)) and $J$ as in (3.3). Recall (see the first paragraph of Sect. 3) that the definition of $L$ does not involve accelerants and depends on (1.10) only. However, if the potential $v$ of (1.10) is given by an accelerant, then Proposition 3.1 tells us that $L$ is similar to the operator of integration with a similarity operator of a special kind. The next proposition goes in the reverse direction.

Proposition 5.3. Assume that the operator $L$ defined by (5.14) is similar to the operator of integration, $L=\Lambda^{-1} A \Lambda$, where $\Lambda$ and $\Lambda^{-1}$ have the following 
properties. Both $\Lambda$ and $\Lambda^{-1}$ are lower triangular operators,

$$
\begin{aligned}
(\Lambda f)(x) & =f(x)+\int_{0}^{x} \rho(x, t) f(s) d s, \quad 0 \leq x \leq \mathbf{T}, \\
\left(\Lambda^{-1} f\right)(x) & =f(x)+\int_{0}^{x} \rho^{\times}(x, s) f(s) d s, \quad 0 \leq x \leq \mathbf{T},
\end{aligned}
$$

with $\rho(x, s)$ and $\rho^{\times}(x, s)$ being continuous $r \times r$ matrix functions on the triangles $0 \leq s \leq x \leq \mathbf{T}$. Furthermore, we assume that $\Lambda$ and $\Lambda^{-1}$ map continuously differentiable functions into continuously differentiable functions, and

$$
\left(\Lambda \theta_{0,1}\right)(0)=\frac{1}{\sqrt{2}} \operatorname{Ir}, \quad \frac{1}{\sqrt{2}}\left(\Lambda^{-1} I_{r}\right)(x)=\theta_{0,2}(x) \quad(0 \leq x \leq \mathbf{T}) .
$$

Then the $r \times r$ matrix function $k$ given by

$$
k(x)= \begin{cases}-\frac{1}{\sqrt{2}} \frac{d}{d x}\left(\Lambda \theta_{0,1}\right)(x), & \text { for } 0<x \leq \mathbf{T}, \\ k(-x)^{*}, & \text { for }-\mathbf{T} \leq x<0 .\end{cases}
$$

is an accelerant and $k$ generates the potential $v$.

The above result will allow us to complete the proof of Theorem 1.2. In fact, using Proposition 4.1, we shall show that given $L$ as above a similarity operator $\Lambda$ with the properties described in Proposition 5.3 always exists.

Proof. Let $k$ be defined by (5.18). Clearly, $k$ is hermitian on $[-\mathbf{T}, \mathbf{T}]$. Since $\theta_{0,1}$ is continuously differentiable, the fact that $\Lambda$ maps continuously differentiable functions into continuously differentiable functions implies that $k$ is continuous on $[-\mathbf{T}, \mathbf{T}]$ with a possible jump discontinuity at the origin. The proof that $k$ is an accelerant and generates the potential $v$ will be split into two parts.

Part 1. In this part we show that $k$ is an accelerant. Let $T_{\tau}$ be the operator on $L_{r}^{2}(0, \tau)$ given by

$$
\left(T_{\tau} f\right)(t)=f(t)-\int_{0}^{\tau} k(t-s) f(s) d s, \quad 0 \leq t \leq \tau .
$$

To prove that $k$ is an accelerant, we have to show that the operator $T_{\mathbf{T}}$ is strictly positive on $L_{r}^{2}(0, \tau)$. To establish the latter fact we prove the following identity:

$$
T=T_{\mathbf{T}}=\Lambda \Lambda^{*} .
$$

Note that the right hand side of (5.20) is an $L U$-factorization.

In order to establish (5.20), recall that $L=\Lambda^{-1} A \Lambda$, where $A$ is the operator of integration. Take $f \in L_{r}^{2}(0, \tau)$. Using the similarity relation 
$L=\Lambda^{-1} A \Lambda$ it follows that

$$
\Lambda^{-1} A \Lambda f+\left(\Lambda^{-1} A \Lambda\right)^{*} f=L f+L^{*} f=\theta(\cdot) J \int_{0}^{\mathbf{T}} \theta(t)^{*} f(t) d t .
$$

By multiplying (5.21) from the left by $\Lambda$ and replacing $f$ by $\Lambda^{*} f$ we obtain

$$
A \Lambda \Lambda^{*} f+\Lambda \Lambda^{*} A^{*} f=(\Lambda \theta)(\cdot) J \int_{0}^{\mathrm{T}}(\Lambda \theta)(t)^{*} f(t) d t .
$$

Thus the selfadjoint operator $S=\Lambda \Lambda^{*}$, which acts on $L_{r}^{2}(0, \tau)$, satisfies the identity

$$
A S f+S A^{*} f=(\Lambda \theta)(\cdot) J \int_{0}^{\mathrm{T}}(\Lambda \theta)(t)^{*} f(t) d t, \quad f \in L_{r}^{2}(0, \tau) .
$$

Now, with $k$ given by (5.18), let $s$ be the $r \times r$ matrix function defined by

$$
s(x)=\frac{1}{2} I_{r}-\int_{0}^{x} k(t) d t \quad 0<x \leq \mathbf{T} .
$$

From the first identity in (5.17) and the definition of $k$ in (5.18) we see that $\Lambda \theta_{0,1}=\sqrt{2} s$. By applying $\Lambda$ to both sides of the second identity in (5.17) we obtain $\Lambda \theta_{0,2}=(\sqrt{2})^{-1} I_{r}$. Summarizing we have

$$
\Lambda \theta=\frac{1}{\sqrt{2}}\left[2 s(\cdot) I_{r}\right] .
$$

Using the later identity in the right hand side of (5.23) we obtain

$$
\begin{aligned}
& (\Lambda \theta)(\cdot) J \int_{0}^{\mathbf{T}}(\Lambda \theta)(t)^{*} f(t) d t \\
& =\frac{1}{2}\left[\begin{array}{ll}
2 s(x) I_{r}
\end{array}\right] J \int_{0}^{\mathbf{T}}\left[\begin{array}{c}
2 s(t)^{*} \\
I_{r}
\end{array}\right] f(t) d t \\
& =\frac{1}{2}\left[2 s(x) I_{r}\right] \int_{0}^{\mathbf{T}}\left[\begin{array}{c}
I_{r} \\
2 s(t)^{*}
\end{array}\right] f(t) d t \\
& =s(x) \int_{0}^{\mathbf{T}} f(t) d t+\int_{0}^{\mathbf{T}} s(t)^{*} f(t) d t, \quad 0 \leq x \leq \mathbf{T} .
\end{aligned}
$$


But then (5.23) can be rewritten as

$$
\left(A S f+S A^{*} f\right)(x)=\int_{0}^{\mathbf{T}}\left(s(x)+s(t)^{*}\right) f(t) d t, \quad 0 \leq x \leq \mathbf{T} .
$$

According to Theorem 2.2 in Chapter 1 of [14] (see also [7] and [12]), the equation (5.26) has a unique solution which is given by

$$
(S f)(x)=\frac{d}{d x} \int_{0}^{\mathbf{T}} s(x-t) f(t) d t, \quad s(-x)=-s(x)^{*} \quad(0<x \leq \mathbf{T}) .
$$

(Note Theorem 2.2 in Chapter 1 of [14] is stated for scalar kernel functions, but the result also holds for matrix-valued kernel functions [13]. In fact, to get the result for matrix-valued kernel functions one just writes $S$ as a $r \times r$ matrix with operator entries and applies the scalar-valued result to each of these entries.) From (5.27) and (5.24) we see that $S=T_{\mathbf{T}}$, and thus (5.20) is proved. In particular, $k$ is an accelerant.

Part 2. Let $\tilde{v}$ be the potential generated by the accelerant $k$, where $k$ is as in the previous part. In this part we show that $v=\tilde{v}$.

Consider the canonical system (1.10) with the potential $v$ being replaced by $\tilde{v}$. Let $\tilde{u}(x, \lambda)$ be the corresponding fundamental solution normalized at $x=0$ by $\tilde{u}(0, \lambda)=Q^{*}$, where $Q$ is as in (2.2). Put

$$
\tilde{\theta}(x)=\left[\begin{array}{ll}
I_{r} & 0
\end{array}\right] \tilde{u}(x, 0), \quad \tilde{\omega}(x)=\left[\begin{array}{ll}
0 & I_{r}
\end{array}\right] \tilde{u}(x, 0 \quad(0 \leq x \leq \mathbf{T}) .
$$

From (5.10) we know that

$$
\tilde{\omega}^{\prime} J \tilde{\theta}^{*}=-i \tilde{v}^{*}, \quad 0 \leq x \leq \mathbf{T} .
$$

Thus to prove $v=\tilde{v}$ it suffices to show that $\theta=\tilde{\theta}$ and $\omega=\tilde{\omega}$.

We first show that $\theta=\tilde{\theta}$. Since $k$ is an accelerant generating the potential $\tilde{v}$, we can apply the results of Sects. 2 and 3 to the canonical system (1.10) with $\tilde{v}$ in place of $v$. In particular, using (3.8) in the present setting, we see that

$$
\tilde{\theta}=\frac{1}{\sqrt{2}} \Lambda^{-1} \tilde{\ell}, \quad \text { where } \tilde{\ell}(x)=\left[I_{r}-2 \int_{0}^{x} k(t) d t I_{r}\right] .
$$

Here $\Lambda$ is the lower triangular integral operator which appears in the $L U$ factorization (5.20) of the convolution operator $T=T_{\mathbf{T}}$ defined by $k$ via (5.19). By (5.24) we have $\tilde{\ell}(x)=\left[\begin{array}{ll}2 s(x) & I_{r}\end{array}\right]$, and hence, using (5.25), we obtain $\tilde{\theta}=\theta$.

Next we prove that $\tilde{\omega}=\omega$. By applying Lemma 5.2 to the canonical system (1.10) with $\tilde{v}$ in place of $v$, we have

$$
\tilde{\theta}(x) J \tilde{\omega}(x)^{*}=0, \quad \tilde{\omega}^{\prime}(x) J \tilde{\omega}(x)^{*}=0, \quad \tilde{\omega}(0)=\frac{1}{2} \sqrt{2}\left[\begin{array}{ll}
-I_{r} & I r
\end{array}\right] .
$$

However, $\tilde{\theta}=\theta$. Thus (5.9) holds with $\tilde{\omega}$ in place of $\omega$. But then we can use the uniqueness statement in Lemma 5.2 to show that $\tilde{\omega}=\omega$. 
We have now proved that $v=\tilde{v}$, and hence $k$ is an accelerant generating the potential $v$.

Completing the proof of Theorem 1.2. Let $L$ be the lower triangular semiseparable integral operator defined by (3.2); see also (5.14). In order to complete the proof of Theorem 1.2 it suffices to show that $L$ is similar to the operator $A$ of integration, $L=\Lambda^{-1} A \Lambda$, where $\Lambda$ has all the properties stated in Proposition 5.3. For this purpose we use Proposition 4.1 with

$$
K=L, \quad F(x)=\theta(x), \quad G(x)=J \theta(x)^{*} \quad(0 \leq x \leq \mathbf{T}) .
$$

By Lemma 5.1, the functions $F$ and $G$ in (5.29) are continuously differentiable on $[0, \mathbf{T}]$ and condition (4.2) is satisfied. Furthermore, the second identity in (5.4) implies that for $F$ and $G$ in (5.29) the solution $\rho$ of the differential equation (4.4) is identically equal to $I_{r}$. Thus, by Proposition 4.1,

$$
L=E A E^{-1},
$$

where $A$ is the operator of integration defined by (3.4) and $E$ on $L_{r}^{2}(0, \mathbf{T})$ is a lower triangular integral operator of the form

$$
(E f)(x)=f(x)+\int_{0}^{x} e(x, t) f(t) d t, \quad f \in L_{r}^{2}(0, \mathbf{T}) .
$$

Moreover, we know that $e(x, t)$ is a continuous $r \times r$ matrix function on $0 \leq t \leq x \leq \mathbf{T}$, which is zero at $t=0$, and the operators $E^{ \pm 1}$ map functions with a continuous derivative into functions with a continuous derivative.

To construct the lower triangular integral operator $\Lambda$ we need (apart from the operator $E$ ) an additional normalizing lower triangular operator. This operator is the lower triangular convolution operator $E_{0}$ defined by

$$
\begin{gathered}
\left(E_{0} f\right)(x)=\theta_{0,2}(0) f(x)+\int_{0}^{x} e_{0}(x-t) f(t) d t, \text { where } \\
e_{0}(x):=\frac{d}{d x}\left(E^{-1} \theta_{0,2}\right)(x) .
\end{gathered}
$$

Recall that $\theta_{0,2}$ is defined by the second identity in (5.12). Since $\theta$ is continuously differentiable (see Lemma 5.1), the same holds true for $\theta_{0,2}$. Using the fact that $E^{-1}$ maps functions with a continuous derivative into functions with a continuous derivative, we conclude that $e_{0}$ is continuous (in fact, continuously differentiable).

Lemma 5.4. Let $E_{0}$ be the operator on $L_{r}^{2}(0, \mathbf{T})$ defined by (5.32), and let $A$ be the operator of integration defined by (3.4). Then

$$
E_{0} A=A E_{0} \quad \text { and } \quad\left(E_{0} I_{r}\right)(x)=\left(E^{-1} \theta_{0,2}\right)(x) \quad(0 \leq x \leq \mathbf{T}) .
$$

Furthermore, $E_{0}$ is invertible and $E_{0}^{ \pm 1}$ map functions with a continuous derivative into functions with a continuous derivative. 
Proof. Since $E_{0}$ is a lower triangular convolution integral operator, $E_{0}$ commutes with the operator of integration. Thus the first identity in (5.34) holds. From (5.31) with $f=\theta_{0,2}$ we see that $\left(E^{-1} \theta_{0,2}\right)(0)=\theta_{0,2}(0)$. By using the latter identity, (5.32), and (5.33) we obtain

$$
\begin{aligned}
\left(E_{0} I_{r}\right)(x) & =\theta_{0,2}(0)+\int_{0}^{x} e_{0}(x-t) d t=\theta_{0,2}(0)+\int_{0}^{x} e_{0}(t) d t \\
& =\theta_{0,2}(0)+\int_{0}^{x} \frac{d}{d t}\left(E^{-1} \theta_{0,2}\right)(t) d t \\
& =\theta_{0,2}(0)+\left(E^{-1} \theta_{0,2}\right)(x)-\theta_{0,2}(0)=\left(E^{-1} \theta_{0,2}\right)(x),
\end{aligned}
$$

which yields the second identity in (5.34). According to (5.2), (5.12), and the initial condition in (5.1), we have

$$
\left[\theta_{0,1}(0) \quad \theta_{0,2}(0)\right]=\left[\begin{array}{ll}
I_{r} & 0
\end{array}\right] u(0,0)=\frac{1}{\sqrt{2}}\left[\begin{array}{ll}
I_{r} & I_{r}
\end{array}\right] .
$$

In particular, $\theta_{0,2}(0)=I_{r} / \sqrt{2}$, and so $E_{0}$ is invertible. Furthermore, $E_{0}^{-1}$ is of the form

$$
\left(E_{0}^{-1} f\right)(x)=\theta_{2}(0)^{-1} f(x)+\int_{0}^{x} e_{0}^{\times}(x-t) f(t) d t, \quad 0 \leq x \leq \mathbf{T},
$$

with $e_{0}^{\times}(x)$ being continuous on $0 \leq x \leq \mathbf{T}$.

Next, let $f$ be any $\mathbb{C}^{r}$-valued function on $[0, \mathbf{T}]$ with a continuous derivative. Write $f$ as $f(\cdot)=(A g)(\cdot)+u$, where $g$ is the derivative of $f$ and $u$ is a constant $r \times r$ matrix. Then $E_{0} f=E_{0} A g+E_{0} u=A E_{0} g+E_{0} u$. Since $e_{0}$ and $g$ are continuous functions, $E_{0} g$ is continuous, and thus $E_{0} A g$ is continuously differentiable. Hence in order to prove that $E_{0} f$ is continuously differentiable, it suffices to show that $E_{0} u$ has this property. The latter can be derived from the second identity in (5.34) and the properties of $E$. A more direct argument is as follows. From (5.32) we see that

$$
\left(E_{0} u\right)(x)=\theta_{2}(0) u+\int_{0}^{x} e_{0}(x-t) u d t=\theta_{2}(0) u+\int_{0}^{x} e_{0}(t) u d t .
$$

Since $e_{0}$ is continuous, this implies that $E_{0} u$ is continuously differentiable as desired. In a similar way, using that $E_{0}^{-1}$ commutes with $A$ and that $E_{0}^{-1}$ is given by (5.36) with $e_{0}^{\times}$being continuous, one shows that $E_{0}^{-1}$ maps functions with a continuous derivative into functions with a continuous derivative.

For latter purposes we note that

$$
\left(E_{0}^{-1} E^{-1} \theta_{0,1}\right)(0)=I_{r} .
$$

To see this, observe that by $(5.36)$ for any continuous $\mathbb{C}^{r}$-valued function $f$ we have $\left(E_{0}^{-1} f\right)(0)=\theta_{0,2}(0)^{-1} f(0)$. We apply this identity to $f=E^{-1} \theta_{0,1}$. We know that $\theta_{0,1}$ is continuously differentiable, and hence $E^{-1} \theta_{0,1}$ has the same property. In particular, $E^{-1} \theta_{0,1}$ is continuous. Using (4.36) and the 
fact that in this case $\rho$ defined by (4.4) is identically equal to $I_{r}$, we see that $\left(E^{-1} \theta_{0,1}\right)(0)=\theta_{0,1}(0)$. Thus

$$
\left(E_{0}^{-1} E^{-1} \theta_{0,1}\right)(0)=\theta_{0,2}(0)^{-1}\left(E^{-1} \theta_{0,1}\right)(0)=\theta_{0,2}(0)^{-1} \theta_{0,1}(0) .
$$

But then (5.35) yields (5.37).

Now define

$$
\Lambda=\frac{1}{\sqrt{2}} E_{0}^{-1} E^{-1} .
$$

We claim that $\Lambda$ given by (5.38) satisfies all the conditions on $\Lambda$ stated in Proposition 5.3. Indeed, from (5.30) and the first identity in (5.34) we see that $L=\Lambda A \Lambda^{-1}$. Furthermore, $\Lambda$ and $\Lambda^{-1}$ are lower triangular integral operators of the form (5.15) and (5.16), respectively, and their respective kernel functions are continuous on the triangles $0 \leq s \leq t \leq \mathbf{T}$, because the kernel functions of $E^{ \pm 1}$ and $E_{0}^{ \pm 1}$ have these properties. Since $E^{ \pm 1}$ and $E_{0}^{ \pm 1}$ map functions with a continuous derivative into functions with a continuous derivative, the same holds true for $\Lambda$ and $\Lambda^{-1}$. It remains to check the identities in (5.17). The first identity in (5.17) follows from the definition of $\Lambda$ in (5.38) and the equality in (5.37). Finally, we use the second equality in (5.34). The latter can be rewritten as $E_{0}^{-1} E^{-1} \theta_{0,2}=I_{r}$. Using definition of $\Lambda$ in (5.38), this yields the second identity in (5.17).

Thus $\Lambda$ given by (5.38) satisfies all the conditions on $\Lambda$ appearing in Proposition 5.3. Hence the potential $v$ is generated by an accelerant, as desired.

\section{Pseudo-Exponential Potentials}

In this section we consider the class of so-called pseudo-exponential potentials, which has been introduced in [4]; see also [5]. The aim is to show how Theorem 2.1 can be used to present an alternative proof of the basic formula for the fundamental solution given in Theorem 4.2 of [4]; see also Section 2 in [5].

We begin with some notation. Fix an integer $n>0$ and a triple of parameter matrices: an $n \times n$ matrix $\mathcal{B}$ and $n \times r$ matrices $\Phi_{1}$ and $\Phi_{2}$. Recall that the triple $\mathcal{B}, \Phi_{1}, \Phi_{2}$ is called admissible whenever

$$
\mathcal{B}^{*}-\mathcal{B}=i \Phi_{2} \Phi_{2}^{*}
$$

Throughout $\Phi$ is the $n \times r$ matrix given by $\Phi=\Phi_{1}+i \Phi_{2}$.

Now let $\mathcal{B}, \Phi_{1}$ and $\Phi_{2}$ be an admissible triple, and put

$$
k(t)=-2 \Phi_{1}^{*} e^{2 i t \mathcal{B}^{*}} \Phi, \quad k(-t)=k(t)^{*}, \quad t>0 .
$$

By taking adjoints, a minor modification of the proof of Proposition 5.2 in [1] shows that the function $k$ is an accelerant on each interval $[-\mathbf{T}, \mathbf{T}]$, and the corresponding potential is given by

$$
v(\tau)=2 i \Phi_{1}^{*} e^{i \tau \mathcal{A}^{*}} \Sigma(\tau)^{-1} e^{i \tau \mathcal{A}} \Phi, \quad \mathcal{A}=\mathcal{B}-\Phi_{1} \Phi_{2}^{*},
$$


where

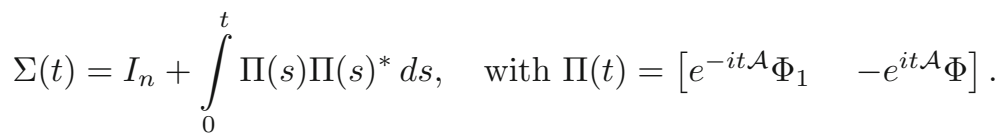

Note that with $\Phi_{1}=\gamma_{1}$ and $\Phi_{2}=\gamma_{2}$, we have $\Phi=\gamma_{1}+i \gamma_{2}$, and in this case $v$ in (6.3) is just equal to $v$ given by (4.6) in [4]. The following result is a variant of Theorem 4.2 in [4].

Proposition 6.1. Let $\mathcal{B}, \Phi_{1}$ and $\Phi_{2}$ be an admissible triple, and let $v$ be the potential defined by (6.3). Then the fundamental solution $u(x, \lambda)$ of the canonical system (1.10) satisfying the initial condition (2.2) is given by

$$
u(\tau, \lambda)=w_{\mathcal{A}, \Pi}(\tau, \lambda) e^{i \tau \lambda j} w_{\mathcal{A}, \Pi}(0, \lambda)^{-1} Q^{*},
$$

where $j$ is the $2 r \times 2 r$ matrix in the left hand side of (1.11) and

$$
w_{\mathcal{A}, \Pi}(\tau, \lambda)=I_{2 r}+i j \Pi(\tau)^{*} \Sigma(\tau)^{-1}\left(\lambda I_{n}-\mathcal{A}\right)^{-1} \Pi(\tau) .
$$

The proof of Proposition 6.1 given below is very different from the proof of Theorem 4.2 in [1]. Here we shall use that the potential $v$ in (6.3) is generated by the accelerant $k$ in (6.2). This fact will allow us to employ the formula for the fundamental solution given in Theorem 2.1.

We shall only prove equality (6.5) for the block $\omega_{2}(\tau, \lambda)$ of $u(\tau, \lambda)$ (see (2.7)); the representation of the other blocks can be proved in a similar way.

Proof. We shall show that $\omega_{2}=\widehat{\omega}_{2}$, where $\widehat{\omega}_{2}$ denotes the right lower block on the right-hand side of (6.5). In Theorem 2.1 the block $\omega_{2}(\tau, \lambda$ is given (cf., (2.9)) by

$$
\omega_{2}(\tau, \lambda)=\frac{1}{\sqrt{2}} e^{-i \tau \lambda}\left\{I_{r}+\int_{0}^{\tau} e^{2 i s \lambda} \gamma_{\tau}(0, s) d s\right\} .
$$

Here $\gamma_{\tau}(t, s)$ is the resolvent kernel corresponding to the accelerant $k$. Using adjoints, the same line of reasoning as in the proof of Proposition 5.2 in [1], shows that

$$
\gamma_{\tau}(0, s)=-2 \Phi^{*} e^{-i \tau \mathcal{A}^{*}} \Sigma(\tau)^{-1} e^{-i \tau \mathcal{A}}\left[\begin{array}{ll}
I_{n} & 0
\end{array}\right] e^{i(s-\tau) A_{M}^{\times}}\left[\begin{array}{l}
\Phi_{1} \\
\Phi_{2}
\end{array}\right],
$$

where

$$
A_{M}^{\times}=-2\left[\begin{array}{cc}
\mathcal{A} & -\Phi_{1} \Phi_{1}^{*} \\
0 & \mathcal{A}^{*}
\end{array}\right] .
$$

In what follows we shall use the identity

$$
\left[\begin{array}{ll}
I_{n} & 0
\end{array}\right] e^{-i \tau A_{M}^{\times}}\left[\begin{array}{c}
I_{n} \\
i I_{n}
\end{array}\right]=e^{i \tau \mathcal{A}} \Sigma(\tau) e^{i \tau \mathcal{A}^{*}} .
$$

Here $A_{M}^{\times}$and $\Sigma(\tau)$ are as in (6.9) and (6.4), respectively. Note that (6.10) is the analogue of formula (4.7) in [4]. 
By substituting (6.8) in (6.7) we get

$$
\begin{aligned}
\omega_{2}(\tau, \lambda)= & \frac{1}{\sqrt{2}} e^{-i \tau \lambda}\left\{I_{r}+2 i \Phi^{*} e^{-i \tau \mathcal{A}^{*}} \Sigma(\tau)^{-1} e^{-i \tau \mathcal{A}}\left[\begin{array}{ll}
I_{n} & 0
\end{array}\right]\right. \\
& \left.\times\left(2 \lambda I_{2 n}+A_{M}^{\times}\right)^{-1}\left(e^{2 i \tau \lambda} I_{2 n}-e^{-i \tau A_{M}^{\times}}\right)\left[\begin{array}{l}
\Phi_{1} \\
\Phi_{2}
\end{array}\right]\right\} .
\end{aligned}
$$

Since $A_{M}^{\times}$is given by (6.9), we can rewrite (6.11) in the form

$$
\begin{aligned}
\omega_{2}(\tau, \lambda)= & \frac{1}{\sqrt{2}} e^{-i \tau \lambda}\left\{I_{r}-i \Phi^{*} e^{-i \tau \mathcal{A}^{*}} \Sigma(\tau)^{-1} e^{-i \tau \mathcal{A}}\left(\mathcal{A}-\lambda I_{n}\right)^{-1}\right. \\
& \left.\times\left[I_{n} \Phi_{1} \Phi_{1}^{*}\left(\mathcal{A}^{*}-\lambda I_{n}\right)^{-1}\right]\left(e^{2 i \tau \lambda} I_{2 n}-e^{-i \tau A_{M}^{\times}}\right)\left[\begin{array}{l}
\Phi_{1} \\
\Phi_{2}
\end{array}\right]\right\} .
\end{aligned}
$$

Partition $e^{-i \tau A_{M}^{\times}}$into $n \times n$ blocks $\left(e^{-i \tau A_{M}^{\times}}\right)_{k j}$. From (6.9) and (6.10) it follows that

$$
\begin{aligned}
& \left(e^{-i \tau A_{M}^{\times}}\right)_{11}=e^{2 i \tau \mathcal{A}}, \quad\left(e^{-i \tau A_{M}^{\times}}\right)_{22}=e^{2 i \tau \mathcal{A}^{*}}, \\
& \left(e^{-i \tau A_{M}^{\times}}\right)_{21}=0, \quad\left(e^{-i \tau A_{M}^{\times}}\right)_{12}=i\left(e^{2 i \tau \mathcal{A}}-e^{i \tau \mathcal{A}} \Sigma(\tau) e^{i \tau \mathcal{A}^{*}}\right) .
\end{aligned}
$$

Taking into account (6.12)-(6.14) we arrive at

$$
\begin{aligned}
\omega_{2}(\tau, \lambda)= & \frac{1}{\sqrt{2}} e^{i \tau \lambda}\left\{-i \Phi^{*} e^{-i \tau \mathcal{A}^{*}} \Sigma(\tau)^{-1} e^{-i \tau \mathcal{A}}\left(\mathcal{A}-\lambda I_{n}\right)^{-1} \Phi_{1}\right. \\
& \left.\times\left(I_{r}+\Phi_{1}^{*}\left(\mathcal{A}^{*}-\lambda I_{n}\right)^{-1} \Phi_{2}\right)\right\}+\frac{1}{\sqrt{2}} e^{-i \tau \lambda} \\
& \times\left\{I_{r}+i \Phi^{*} e^{-i \tau \mathcal{A}^{*}} \Sigma(\tau)^{-1} e^{-i \tau \mathcal{A}}\left(\mathcal{A}-\lambda I_{n}\right)^{-1}\left(e^{2 i \tau \mathcal{A}} \Phi_{1}\right.\right. \\
& \left.\left.+\Phi_{1} \Phi_{1}^{*}\left(\mathcal{A}^{*}-\lambda I_{n}\right)^{-1} e^{2 i \tau \mathcal{A}^{*}} \Phi_{2}+i\left(e^{2 i \tau \mathcal{A}}-e^{i \tau \mathcal{A}} \Sigma(\tau) e^{i \tau \mathcal{A}^{*}}\right) \Phi_{2}\right)\right\} .
\end{aligned}
$$

Now, consider the right lower block $\widehat{\omega}_{2}$ of the right-hand side of (6.5). The transfer matrix function $w_{\mathcal{A}, \Pi}(\tau, \lambda)$ has the property (see, e.g., [15]):

$$
w_{\mathcal{A}, \Pi}(\tau, \bar{\lambda})^{*} j w_{\mathcal{A}, \Pi}(\tau, \lambda)=j .
$$

In particular, we have $w_{\mathcal{A}, \Pi}(0, \lambda)^{-1}=j w_{\mathcal{A}, \Pi}(0, \bar{\lambda})^{*} j$. Hence, using (2.2), (6.4), and (6.6), we can write

$$
\begin{aligned}
& \widehat{\omega}_{2}(\tau, \lambda) \\
&=\frac{1}{\sqrt{2}}\left(\left[\begin{array}{ll}
0 & I_{r}
\end{array}\right]-i \Phi^{*} e^{-i \tau \mathcal{A}^{*}} \Sigma(\tau)^{-1}\left(\mathcal{A}-\lambda I_{n}\right)^{-1}\left[e^{-i \tau \mathcal{A}} \Phi_{1}\right.\right.\left.\left.-e^{i \tau \mathcal{A}} \Phi\right]\right) \\
& \times e^{i \tau \lambda j}\left(I_{2 r}+i j\left[\begin{array}{c}
\Phi_{1}^{*} \\
-\Phi^{*}
\end{array}\right]\left(\mathcal{A}^{*}-\lambda I_{n}\right)^{-1}\left[\begin{array}{ll}
\Phi_{1} & -\Phi
\end{array}\right]\right)\left[\begin{array}{c}
I_{r} \\
I_{r}
\end{array}\right] .
\end{aligned}
$$


Formula (6.15) has the form

$$
\omega_{2}(\tau, \lambda)=\frac{1}{\sqrt{2}} e^{i \tau \lambda} c_{+}(\tau, \lambda)+\frac{1}{\sqrt{2}} e^{-i \tau \lambda} c_{-}(\tau, \lambda),
$$

where $c_{ \pm}$are the expressions between curly braces contained in (6.15). Formula (6.16) can be rewritten in a similar form

$$
\widehat{\omega}_{2}(\tau, \lambda)=\frac{1}{\sqrt{2}} e^{i \tau \lambda \widehat{c}_{+}}(\tau, \lambda)+\frac{1}{\sqrt{2}} e^{-i \tau \lambda \widehat{c}_{-}}(\tau, \lambda),
$$

where

$$
\begin{aligned}
& \widehat{c}_{+}(\tau, \lambda)=-i \Phi^{*} e^{-i \tau \mathcal{A}^{*}} \Sigma(\tau)^{-1}\left(\mathcal{A}-\lambda I_{n}\right)^{-1} e^{-i \tau \mathcal{A}} \Phi_{1} \\
& \times\left(I_{r}+\Phi_{1}^{*}\left(\mathcal{A}^{*}-\lambda I_{n}\right)^{-1} \Phi_{2}\right), \\
& \widehat{c}_{-}(\tau, \lambda)=\left(I_{r}+i \Phi^{*} e^{-i \tau \mathcal{A}^{*} \Sigma(\tau)^{-1}}\left(\mathcal{A}-\lambda I_{n}\right)^{-1} e^{i \tau \mathcal{A}} \Phi\right) \\
& \times\left(I_{r}+\Phi^{*}\left(\mathcal{A}^{*}-\lambda I_{n}\right)^{-1} \Phi_{2}\right) .
\end{aligned}
$$

In (6.19) and (6.20) we used the equality $\Phi_{1}-\Phi=-i \Phi_{2}$; see the second paragraph of this section. Comparing (6.15) and (6.19) yields $c_{+}=\widehat{c}_{+}$. To prove that $c_{-}=\widehat{c}_{-}$we shall need the equality $\mathcal{A} \Sigma(\tau)-\Sigma(\tau) \mathcal{A}^{*}=i \Pi(\tau) j \Pi(\tau)^{*}$, that is, equality (1.22) from [4] rewritten in our present notations. Equivalently, we have

$$
\begin{aligned}
& \Sigma(\tau)\left(\mathcal{A}^{*}-\lambda I_{n}\right)+i e^{-i \tau \mathcal{A}} \Phi_{1} \Phi_{1}^{*} e^{i \tau \mathcal{A}^{*}}-i e^{i \tau \mathcal{A}} \Phi \Phi^{*} e^{-i \tau \mathcal{A}^{*}} \\
& \quad=\left(\mathcal{A}-\lambda I_{n}\right) \Sigma(\tau) .
\end{aligned}
$$

Now, use $(6.15),(6.20)$, and $e^{2 i \tau \mathcal{A}}\left(\Phi_{1}+i \Phi_{2}\right)=e^{2 i \tau \mathcal{A}} \Phi$ to get

$$
\begin{aligned}
\widehat{c}_{-}(\tau, & \lambda)-c_{-}(\tau, \lambda)=\Phi^{*}\left(\mathcal{A}^{*}-\lambda I_{n}\right)^{-1} \Phi_{2} \\
& -i \Phi^{*} e^{-i \tau \mathcal{A}^{*}} \Sigma(\tau)^{-1}\left(\mathcal{A}-\lambda I_{n}\right)^{-1} \\
& \times\left(-e^{i \tau \mathcal{A}} \Phi \Phi^{*} e^{-i \tau \mathcal{A}^{*}}+e^{-i \tau \mathcal{A}} \Phi_{1} \Phi_{1}^{*} e^{i \tau \mathcal{A}^{*}}-i \Sigma(\tau)\left(\mathcal{A}^{*}-\lambda I_{n}\right)\right) \\
& \times e^{i \tau \mathcal{A}^{*}}\left(\mathcal{A}^{*}-\lambda I_{n}\right)^{-1} \Phi_{2} .
\end{aligned}
$$

Finally, we substitute (6.21) into (6.22). This yields $\widehat{c}_{-}(\tau, \lambda)=c_{-}(\tau, \lambda)$. Hence we have $\widehat{c}_{ \pm}=c_{ \pm}$, and formulas (6.17) and (6.18) imply $\omega_{2}=\widehat{\omega}_{2}$.

Open Access. This article is distributed under the terms of the Creative Commons Attribution Noncommercial License which permits any noncommercial use, distribution, and reproduction in any medium, provided the original author(s) and source are credited.

\section{References}

[1] Alpay, D., Gohberg, I., Kaashoek, M.A., Lerer, L., Sakhnovich, A.L.: Krein systems. In: Modern Analysis and Applications. The Mark Krein Centenary Conference, vol. 2, OT 191, pp. 19-36. Birkhäuser, Basel (2009)

[2] Gohberg, I., Goldberg, S., Kaashoek, M.A.: Basic Classes of Linear Operators. Birkhäuser, Basel (2003) 
[3] Gohberg, I., Goldberg, S., Kaashoek, M.A.: Classes of Linear Operators, vol. I. Birkhäuser, Basel (1990)

[4] Gohberg, I., Kaashoek, M.A., Sakhnovich, A.L.: Canonical sytems with rational spectral densities: explicit formulas and applications. Math. Nach. 194, 93-125 (1998)

[5] Gohberg, I., Kaashoek, M.A., Sakhnovich, A.L.: Scattering problems for a canonical system with a pseudo-exponential potential. Asymptotic Anal. 29, 1-38 (2002)

[6] Gohberg, I., Koltracht, I.: Numerical solution of integral equations, fast algorithms and Krein-Sobolev equations. Numer. Math. 47, 237-288 (1985)

[7] Koltracht, I., Kon, B., Lerer, L.: Inversion of structured operators. Integral Equ. Oper. Theory 20, 410-480 (1994)

[8] Krein, M.G.: On the theory of accelerants and $S$-matrices of canonical differential systems. Dokl. Akad. Nauk SSSR (N.S.) 111, 1167-1170 (1956)

[9] Sakhnovich, A.L.: Asymptotic behavior of spectral functions of an S-node. Soviet Math. (Iz. VUZ) 32, 92-105 (1988)

[10] Sakhnovich, A.L.: Dirac type and canonical systems: spectral and Weyl-Titchmarsh functions, direct and inverse problems. Inverse Probl. 18, 331-348 (2002)

[11] Sakhnovich, L.A.: Spectral analysis of Volterra's operators defined in the space of vector-functions $L_{m}^{2}(0, l)$. Ukr. Mat. J. 16, 259-268 (1964)

[12] Sakhnovich, L.A.: Equations with a difference kernel on a finite interval. Russian Math. Surv. 35, 81-152 (1980)

[13] Sakhnovich, L.A.: Systems of equations with difference kernels. Ukr. Math. J. 32, 44-50 (1980)

[14] Sakhnovich, L.A.: Integral equations with difference kernels on finite intervals. OT 84. Birkhäuser, Basel (1996)

[15] Sakhnovich, L.A.: Spectral Theory of Canonical Differential Systems. Method of Operator Identities, OT 107. Birkhäuser, Basel (1999)

D. Alpay

Department of Mathematics

Ben-Gurion University of the Negev

Beer-Sheva 84105

Israel

e-mail: dany@math.bgu.ac.il

I. Gohberg (Z"L), M. A. Kaashoek $(\bowtie)$

Afdeling Wiskunde

Faculteit der Exacte Wetenschappen

VU University Amsterdam

De Boelelaan 1081a, 1081 HV Amsterdam, The Netherlands

e-mail: ma.kaashoek@few.vu.nl

L. Lerer

Department of Mathematics

Technion, Israel Institute of Technology

Haifa 32000, Israel

e-mail: llerer@techunix.technion.ac.il 


\begin{abstract}
A. L. Sakhnovich
Fakultät für Mathematik

Universität Wien

Nordbergstrasse 15, 1090 Vienna, Austria

e-mail: al_sakhnov@yahoo.com
\end{abstract}

Received: December 23, 2009.

Revised: May 1, 2010. 